\title{
LOS DESTRUCTORES DE LA CLASE CANNON EN LA MARINA DE GUERRA DEL PERÚ
}

\author{
THE DESTRUCTORS OF THE CANNON CLASS IN THE MARINA DE \\ GUERRA DEL PERÚ
}

\author{
Ernesto Piero Bazzetti De los Santos \\ Universidad Ricardo Palma \\ piero.bazzetti@urp.edu.pe
}

\begin{abstract}
RESUMEN
A raíz de una conferencia, en la que se tocaron algunos temas relacionados a unidades navales que sirvieron en nuestra armada, recibí la propuesta de investigar sobre unidades navales, yo sabía que Jorge Ortiz Sotelo, había estudiado brillantemente el historial de los submarinos peruanos y John Rodríguez Asti; los cruceros; cuestión que no podía repetir, así que empecé esta investigación sobre los destructores en la Marina de Guerra del Perú. En esta primera entrega veremos el historial de los destructores escolta de la clase CANNON. Veremos una breve introducción sobre estas unidades, los buques que sirvieron en diversas armadas, destacando las sudamericanas, también sus especificaciones técnicas, para luego pasar a su historial en la armada estadounidense, su incorporación e historial en nuestra armada, las unidades que en la actualidad se han preservado.
\end{abstract}

PALABRAS CLAVE

destructor, armada, escolta, submarino, crucero.

\begin{abstract}
Following a conference, which touched on some issues related to naval units that served in our Navy, I received the proposal to investigate naval units, I knew that Jorge Ortiz Sotelo, had studied brilliantly the history of Peruvian submarines and John Rodríguez Asti; cruises; I could not repeat, so I started this investigation about the destroyers in the Peruvian Navy. In this first installment, we will see the history of the escort destroyers of the CANNON class. We will see a brief introduction about these units, the ships that served in different navies, highlighting the South American ones, also their technical specifications, to later pass to their history in the US Navy, their incorporation and history in our Navy, the units that currently they have been preserved.
\end{abstract}

\section{KEYWORDS}

destroyer, armed, escort, submarine, cruise. 


\section{HISTORIA Y NOTAS}

La Cannon era una clase de destructores de escolta construida por los Estados Unidos para la escolta de convoyes y la guerra antisubmarina durante la Segunda Guerra Mundial. Fueron construidos entre 1943 y 1944, el buque que dio inicio a esta clase fue el USS CANNON (DE- $_{1}$ 99), comisionado el 26 de septiembre de 1943 en los astilleros Dravo Corporation de Wilmington, Delaware.

La Cannon fue la tercera clase consecutiva de destructores de escolta estadounidenses después de las clases Evarts y Buckley de los que difería principalmente en el sistema de propulsión.

El casco de los destructores de la clase Cannon se basaba en el de la clase Buckley. Las unidades de esta clase, eran más ligeras que los destructores regulares, porque fueron diseñados para realizar tareas de segunda línea, tenían un tamaño mayor al de las fragatas y corbetas regulares, su velocidad máxima era de 21 nudos, no contaban con armamento pesado y estuvieron equipados con motores menos potentes ya que su objetivo era no llegar a la velocidad máxima de alrededor de 30 nudos de las unidades de combate, sino tener una velocidad menor para escoltar a los convoyes.

La propulsión era conocida como del tipo DET (Diesel Electric Tándem).La propulsión tipo DET reemplazó a la planta de propulsión turboeléctrica (TE) y fue la principal diferencia con los destructores de la clase Buckley, la clase que precedió a los Cannon. Tenía cuatro motores diesel con una potencia total de $6000 \mathrm{hp}$, que accionaban los generadores que producían electricidad para los motores eléctricos en tándem. Este sistema fue diseñado para proporcionar a los buques una velocidad máxima de 21 nudos, pero en la práctica no excedían los 18 nudos. A su vez, la propulsión DET fue sustituida por una planta diesel de transmisión directa (FMR) en el diseño de la siguiente clase de destructores, la clase Edsall.

La capacidad interna de combustible era de 315 toneladas, el radio de acción de 10800 millas a una velocidad económica de 12 nudos. La tripulación la componían normalmente 15 oficiales y 201 suboficiales y marineros. 
Con respecto al armamento este consistía en tres cañones de $76 \mathrm{~mm}$ en montajes simples; dos cañones de $40 \mathrm{~mm}$ en un montaje doble; ocho cañones de $20 \mathrm{~mm}$ en montajes simples; un lanzador triple para torpedos de $533 \mathrm{~mm}$; un lanzador de cohetes antisubmarinos; ocho lanzadores de cargas de profundidad y dos lanzaderas a popa para cargas de profundidad.

La electrónica de los destructores de la clase Cannon estaba compuesta por un radar de búsqueda en superficie, un radar de búsqueda aérea, un sistema de control de tiro y un sonar.

De los 116 barcos ordenados, 44 fueron cancelados, por lo que finalmente se construyeron 72. Durante la guerra seis naves fueron entregadas directamente a las fuerzas navales de Francia Libre, en donde se les denominó como clase Sénégalais, a diferencia de los destructores estadounidenses, los franceses carecieron del lanzador de torpedos. Y ocho unidades en servicio con la armada estadounidense fueron transferidas a la Marinha do Brasil. Finalizada la contienda las unidades permanecieron en servicio tanto en la Marine Nationale como en la Marinha do Brasil. Ninguno de los destructores de la clase Cannon se perdió en la guerra.

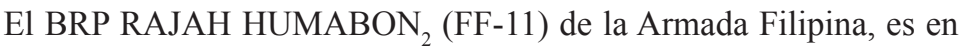
la actualidad el único buque de esta clase en servicio activo en el mundo.

\section{LOS OTROS CANNON}

Terminada la guerra muchos destructores Cannon fueron transferidos a doce armadas, incluyendo nuestra Marina de Guerra del Perú, como parte del Programa de Asistencia Mutua de Defensa (MDAP). Si hacemos un rápido análisis, de las 72 naves construidas, 53 pasaron a formar parte de doce armadas, por lo que solo 19 quedaron en los Estados Unidos siendo su final el desguace. Aquí presentamos unos cuadros en donde aparecen los países que incorporaron destructores de la clase Cannon en sus armadas. Para un mejor entendimiento los hemos separado por continentes.

Cuatro armadas europeas recibieron destructores de la clase Cannon. Como hemos mencionado anteriormente, durante la guerra la armada de la Francia Libre recibió seis unidades que fueron: 


\begin{tabular}{|l|c|l|c|}
\hline \multicolumn{1}{|c|}{ UNIDAD } & \multicolumn{1}{c|}{ N $^{\circ}$} & \multicolumn{1}{c|}{ ANTERIORMENTE } & N $^{\circ}$ \\
\hline ALGÉRIEN & F701 & USS CRONIN & DE-107 \\
\hline SÉNÉGALAIS & F702 & USS CORBESIER & DE-106 \\
\hline SOMALI & F703 & USS SOMALI & DE-111 \\
\hline HOVA & F704 & USS HOVA & DE-110 \\
\hline MAROCAIN & F705 & USS MAROCAIN & DE-109 \\
\hline TUNISIEN & F706 & USS CROSLEY & DE-108 \\
\hline
\end{tabular}

Finalizada la Segunda Guerra Mundial, la Marine Nationale recibió ocho unidades adicionales, lo que convierte a Francia en el mayor usuario de destructores de la clase Cannon con catorce naves en total, estas unidades fueron:

\begin{tabular}{|l|c|l|c|}
\hline \multicolumn{1}{|c|}{ UNIDAD } & \multicolumn{1}{c|}{$\mathbf{N}^{\circ}$} & \multicolumn{1}{c|}{ ANTERIORMENTE } & $\mathbf{N}^{\circ}$ \\
\hline ARABE & F717 & USS SAMUEL S. MILES & DE-183 \\
\hline KABYLE & F718 & USS RIDDLE & DE-185 \\
\hline BAMBARA & F719 & USS SWEARER & DE-186 \\
\hline SAKALAVE & F720 & USS WINGFIELD & DE-194 \\
\hline TOUAREG & F721 & USS BRIGHT & DE-747 \\
\hline SOUDANAIS & F722 & USS CATES & DE-763 \\
\hline BERBÉRE & F723 & USS CLARENCE L. EVANS & DE-113 \\
\hline MALGACHE & F724 & USS BAKER & DE-190 \\
\hline
\end{tabular}

La Armada Real de los Países Bajos recibió seis unidades que fueron:

\begin{tabular}{|l|l|l|l|}
\hline UNIDAD & N $^{\circ}$ & ANTERIORMENTE & $\mathbf{N}^{\circ}$ \\
\hline VAN AMSTEL & F806 & USS BURROWS & DE-105 \\
\hline BITTER & F807 & USS RINEHART & DE-196 \\
\hline VAN EWIJCK & F808 & USS GUSTAFSON & DE-182 \\
\hline DUBOIS & F809 & USS O’NEILL & DE-188 \\
\hline ZEEUW & F810 & USS EISNER & DE-192 \\
\hline VAN ZIJLL & F811 & USS STERN & DE-187 \\
\hline
\end{tabular}


La Armada Griega recibió cuatro unidades que fueron:

\begin{tabular}{|l|c|l|c|}
\hline \multicolumn{1}{|c|}{ UNIDAD } & $\mathbf{N}^{\circ}$ & \multicolumn{1}{|c|}{ ANTERIORMENTE } & \multicolumn{1}{c|}{$\mathbf{N}^{\circ}$} \\
\hline AETOS & D01 & USS SLATER & DE-766 \\
\hline IERAX & D31 & USS EBERT & DE-768 \\
\hline LEON & D54 & USS ELDRIDGE & DE-173 \\
\hline PANTHIR & D67 & USS GARFIELD THOMAS & DE-193 \\
\hline
\end{tabular}

La Marina Militare italiana recibió tres unidades que fueron:

\begin{tabular}{|l|c|l|c|}
\hline \multicolumn{1}{|c|}{ UNIDAD } & \multicolumn{1}{c|}{$\mathbf{N}^{\circ}$} & \multicolumn{1}{|c|}{ ANTERIORMENTE } & \multicolumn{1}{c|}{$\mathbf{N}^{\circ}$} \\
\hline ALDEBARÁN & F-590 & USS THORNHILL & DE-195 \\
\hline ALTAIR & F-591 & USS GANDY & DE-764 \\
\hline ANDRÓMEDA & F-592 & USS WESSON & DE-184 \\
\hline
\end{tabular}

También cinco armadas asiáticas recibieron esta clase buques. La Armada de la República China recibió cuatro unidades que fueron:

\begin{tabular}{|l|c|l|c|}
\hline \multicolumn{1}{|c|}{ UNIDAD } & \multicolumn{1}{c|}{$\mathbf{N}^{\circ}$} & \multicolumn{1}{|c|}{ ANTERIORMENTE } & $\mathbf{N}^{\circ}$ \\
\hline TAI HO & DE-23 & USS THOMAS & DE-102 \\
\hline TAI CHONG & DE-24 & USS BREEMAN & DE-104 \\
\hline TAI HU & DE-25 & USS BOSTWICK & DE-103 \\
\hline TAI CHAO & DE-26 & USS CARTER & DE-112 \\
\hline
\end{tabular}

La Fuerza Marítima de Autodefensa del Japón recibió dos unidades, una de ellas, el HAYSUHI sería transferido a la Armada Filipina, las unidades japonesas fueron:

\begin{tabular}{|l|c|l|c|}
\hline \multicolumn{1}{|c|}{ UNIDAD } & $\mathbf{N}^{\circ}$ & \multicolumn{1}{c|}{ ANTERIORMENTE } & $\mathbf{N}^{\circ}$ \\
\hline ASAHI & DE-262 & USS AMICK & DE-168 \\
\hline HAYSUHI & DE-263 & USS ATHERTON & DE-169 \\
\hline
\end{tabular}

La Armada de la República de Corea recibió dos unidades, ambas 
serían transferidas a la Armada Filipina que los canibalizó y utilizó como fuente de repuestos, los buques coreanos fueron:

\begin{tabular}{|l|c|l|c|}
\hline \multicolumn{1}{|c|}{ UNIDAD } & \multicolumn{1}{|c|}{$\mathbf{N}^{\circ}$} & \multicolumn{1}{|c|}{ ANTERIORMENTE } & N $^{\circ}$ \\
\hline KYONG KI & F-71 & USS MUIR & DE-770 \\
\hline KANG WON & F-72 & USS SUTTON & DE-771 \\
\hline
\end{tabular}

La Real Armada de Tailandia recibió una unidad, que fue:

\begin{tabular}{|l|c|l|c|}
\hline \multicolumn{1}{|c|}{ UNIDAD } & $\mathbf{N}^{\circ}$ & \multicolumn{1}{|c|}{ ANTERIORMENTE } & $\mathbf{N}^{\circ}$ \\
\hline PIN KAO & DE-1 & USS HEMMINGER & DE-746 \\
\hline
\end{tabular}

Por último la Armada Filipina recibió originalmente dos unidades, como ya hemos indicado, más tarde recibió una unidad japonesa y las dos coreanas que se canibalizaron, por lo que los buques filipinos fueron en total cinco:

\begin{tabular}{|l|c|l|c|}
\hline \multicolumn{1}{|c|}{ UNIDAD } & \multicolumn{1}{c|}{$\mathbf{N}^{\circ}$} & \multicolumn{1}{c|}{ ANTERIORMENTE } & $\mathbf{N}^{\circ}$ \\
\hline DATU SIKATUNA & PF-5 & USS AMICK & DE-168 \\
\hline RAJAH HUMABON & PF-11 & HAYSUHI & DE-263 \\
\hline DATU KALANTIAW & PS-76 & USS BOOTH & DE-170 \\
\hline & & KYONG KI & F-71 \\
\hline & & KANG WON & F-72 \\
\hline
\end{tabular}

Tres países sudamericanos también recibieron buques de la clase Cannon. Como ya lo mencionáramos, la Marinha do Brasil recibió ocho unidades durante la guerra, estas unidades fueron:

\begin{tabular}{|l|l|l|c|}
\hline \multicolumn{1}{|c|}{ UNIDAD } & \multicolumn{1}{|c|}{$\mathbf{N}^{\circ}$} & \multicolumn{1}{|c|}{ ANTERIORMENTE } & $\mathbf{N}^{\circ}$ \\
\hline BERTIOGA & Be1 & USS PENNEWILL & DE-175 \\
\hline BEBERIBE & Be2 & USS HERZOG & DE-178 \\
\hline BRACUÍ & Be3 & USS REYBOLD & DE-177 \\
\hline BAURU & Be4 & USS McANN & DE-179 \\
\hline
\end{tabular}




\begin{tabular}{|l|l|l|c|}
\hline BAEPENDI & Be5 & USS CANNON & DE-99 \\
\hline BENEVENTE & Be6 & USS CHRISTOPHER & DE-100 \\
\hline BABITONGA & Be7 & USS ALGER & DE-101 \\
\hline BOCAINA & Be8 & USS MARTS & DE-174 \\
\hline
\end{tabular}

La Armada Nacional de Uruguay recibió dos unidades que fueron:

\begin{tabular}{|l|c|l|c|}
\hline \multicolumn{1}{|c|}{ UNIDAD } & $\mathbf{N}^{\circ}$ & \multicolumn{1}{|c|}{ ANTERIORMENTE } & $\mathbf{N}^{\circ}$ \\
\hline URUGUAY & DE-1 & USS BARON & DE-166 \\
\hline ARTIGAS & DE-2 & USS BRONSTEIN & DE-189 \\
\hline
\end{tabular}

Y por último, la Marina de Guerra del Perú recibió tres unidades, que son el motivo del presente trabajo, las unidades peruanas fueron:

\begin{tabular}{|l|l|l|c|}
\hline \multicolumn{1}{|c|}{ UNIDAD } & $\mathbf{N}^{\circ}$ & \multicolumn{1}{|c|}{ ANTERIORMENTE } & \multicolumn{1}{c|}{$\mathbf{N}^{\circ}$} \\
\hline CASTILLA & D-1 & USS BANGUST & DE-739 \\
\hline AGUIRRE & D-2 & USS WATERMAN & DE-740 \\
\hline RODRÍGUEZ & D-3 & USS WEAVER & DE-741 \\
\hline
\end{tabular}

Por lógicas razones no podemos analizar a todas las unidades transferidas a las doce armadas anteriormente mencionadas, pero si lo haremos brevemente con los buques transferidos a las armadas de nuestra región, es decir las sudamericanas, con la finalidad de saber cuál fue el historial de las naves hermanas de nuestras unidades.

\section{LOS CANNON BRASILEÑOS}

El Bertioga (Be1). Originalmente USS Pennewill (DE-175) de la armada estadounidense. Fue puesto en quilla el 26 de abril de 1943, lanzado el 08 de agosto de 1943 y comisionado el 15 de septiembre de 1943 en el astillero Federal Shipbuilding and Drydock Company de Newark, Nueva Jersey. Su primer comandante fue el teniente John Edward Allen del USNR. Descomisionado y transferido a la Marinha do Brasil el 01 de agosto de 1944 en donde se les denominó contratorpedeiro. Su primer comandante fue el capitán de corbeta José Pereira da Cotta Filho. El 30 de junio de 
1953 pasó nuevamente al US Navy, solo para ser transferido formalmente a la Marinha do Brasil por el Programa de Asistencia Mutua de Defensa (MDAP). Su nombre fue retirado del Registro Naval de Buques de los Estados Unidos en 20 de julio de 1953. En 1955 se cambia su número de casco a D21. En 1960 fue reclasificado como Aviso Oceánico, retirándole todo el armamento antisubmarino y cambiando su número de casco a U26. Fue dado de baja en 1964 y vendido para su desguace en 1975.

El Beberibe (Be2). Originalmente USS Herzog (DE-178) de la armada estadounidense. Fue puesto en quilla el 17 de mayo de 1943, lanzado el 05 de septiembre de 1943 y comisionado el 06 de octubre de 1943 en el astillero Federal Shipbuilding and Drydock Company de Newark, Nueva Jersey. Su primer comandante fue el teniente comandante J. C. Toft Jr. Descomisionado y transferido a la Marinha do Brasil el 01 de agosto de 1944 en donde se les denominó contratorpedeiro. Su primer comandante fue el capitán de corbeta Mário Pinto de Oliveira. El 30 de junio de 1953 pasó nuevamente al US Navy, solo para ser transferido formalmente a la Marinha do Brasil por el Programa de Asistencia Mutua de Defensa (MDAP). Su nombre fue retirado del Registro Naval de Buques de los Estados Unidos en 20 de julio de 1953. En 1955 se cambia su número de casco a D29. En 1960 fue reclasificado como Aviso Oceánico, retirándole todo el armamento antisubmarino y cambiando su número de casco a U29. Encalló el 7 de marzo de 1966 en Ilha Trinidade. Fue dado de baja y vendido para su desguace en 1968.

El Bracuí (Be3). Originalmente USS Reybold (DE-177) de la armada estadounidense. Fue puesto en quilla el 03 de mayo de 1943, lanzado el 22 de agosto de 1943 y comisionado el 29 de septiembre de 1943 en el astillero Federal Shipbuilding and Drydock Company de Newark, Nueva Jersey. Su primer comandante fue el teniente comandante A. B. Bradley Jr. Descomisionado y transferido a la Marinha do Brasil el 15 de agosto de 1944 en donde se les denominó contratorpedeiro. Su primer comandante fue el capitán de corbeta Jorge Carvalhal. El 30 de junio de 1953 pasó nuevamente al US Navy, solo para ser transferido formalmente a la Marinha do Brasil por el Programa de Asistencia Mutua de Defensa (MDAP). Su nombre fue retirado del Registro Naval de Buques de los Estados Unidos en 20 de julio de 1953. En 1955 se cambia su número de casco a D23. En 1960 fue reclasificado como Aviso Oceánico, retirándole todo el armamento 
antisubmarino y cambiando su número de casco a U31. Fue dado de baja el 11 de julio de 1972 y vendido para su desguace.

El Bauru (Be4). Originalmente USS McAnn (DE-179) de la armada estadounidense. Fue puesto en quilla el 17 de mayo de 1943, lanzado el 05 de septiembre de 1943 y comisionado el 11 de octubre de 1943 en el astillero Federal Shipbuilding and Drydock Company de Newark, Nueva Jersey. Su primer comandante fue el comandante Charles F. Hooper. Descomisionado y transferido a la Marinha do Brasil el 15 de agosto de 1944 en donde se les denominó contratorpedeiro. Su primer comandante fue el capitán de corbeta Sylvio Borges de Souza Motta. El 30 de junio de 1953 pasó nuevamente al US Navy, solo para ser transferido formalmente a la Marinha do Brasil por el Programa de Asistencia Mutua de Defensa (MDAP). Su nombre fue retirado del Registro Naval de Buques de los Estados Unidos en 20 de julio de 1953. En 1955 se cambia su número de casco a D18. El 5 de junio de 1964 fue reclasificado como Aviso Oceánico, retirándole todo el armamento antisubmarino y cambiando su número de casco a U28. Fue dado de baja el 17 de septiembre de 1981. El 21 de julio de 1982 se convierte en buque museo, hoy se encuentra preservado en el Centro Cultural da Marinha de Rio de Janeiro. El lector puede ampliar la información sobre esta unidad, porque tenemos desarrollado un capítulo completo, más adelante, sobre los destructores de la clase Cannon preservados hasta nuestros días.

El Baependi (Be5). Originalmente USS Cannon (DE-99) de la armada estadounidense. Fue puesto en quilla el 14 de noviembre de 1942, lanzado el 25 de mayo de 1943 y comisionado el 29 de septiembre de 1943 en el astillero Dravo Corporation de Wilmington, Delaware. Su primer comandante fue el teniente comandante G. Morris. Descomisionado y transferido a la Marinha do Brasil el 19 de diciembre de 1944 en donde se les denominó contratorpedeiro. Su primer comandante fue el capitán de corbeta Raimundo da Costa Figueira. El 30 de junio de 1953 pasó nuevamente al US Navy, solo para ser transferido formalmente a la Marinha do Brasil por el Programa de Asistencia Mutua de Defensa (MDAP). Su nombre fue retirado del Registro Naval de Buques de los Estados Unidos en 20 de julio de 1953. En 1955 se cambia su número de casco a D17. En 1965 fue reclasificado como Aviso Oceánico, retirándole todo el armamento antisubmarino y cambiando su número de casco a U27. Fue dado de baja en 1973 y vendido para su desguace en 1974. 
El Benevente (Be6). Originalmente USS Christopher (DE-100) de la armada estadounidense. Fue puesto en quilla el 07 de diciembre de 1942, lanzado el 19 de junio de 1943 y comisionado el 23 de octubre de 1943 en el astillero Dravo Corporation de Wilmington, Delaware. Su primer comandante fue el teniente W. P. Trench. Descomisionado y transferido a la Marinha do Brasil el 19 de diciembre de 1944 en donde se les denominó contratorpedeiro. Su primer comandante fue el capitán de corbeta Jorge Campello Mauricío de Abreu. El 30 de junio de 1953 pasó nuevamente al US Navy, solo para ser transferido formalmente a la Marinha do Brasil por el Programa de Asistencia Mutua de Defensa (MDAP). Su nombre fue retirado del Registro Naval de Buques de los Estados Unidos en 20 de julio de 1953. En 1955 se cambia su número de casco a D20. En 1960 fue reclasificado como Aviso Oceánico, retirándole todo el armamento antisubmarino y cambiando su número de casco a U30. Fue dado de baja y vendido para su desguace en 1964.

El Babitonga (Be7). Originalmente USS Alger (DE-101) de la armada estadounidense. Fue puesto en quilla el 02 de enero de 1943, lanzado el 08 de julio de 1943 y comisionado el 12 noviembre de 1943 en el astillero Dravo Corporation de Wilmington, Delaware. Su primer comandante fue el teniente comandante W. F. Porter. Descomisionado y transferido a la Marinha do Brasil el 10 de marzo de 1945 en donde se les denominó contratorpedeiro. Su primer comandante fue el capitán de corbeta Daniel Dos Santos Parreira. El 30 de junio de 1953 pasó nuevamente al US Navy, solo para ser transferido formalmente a la Marinha do Brasil por el Programa de Asistencia Mutua de Defensa (MDAP). Su nombre fue retirado del Registro Naval de Buques de los Estados Unidos en 20 de julio de 1953. En 1955 se cambia su número de casco a D16. En 1960 fue reclasificado como Aviso Oceánico, retirándole todo el armamento antisubmarino y cambiando su número de casco a U29. Fue dado de baja y vendido para su desguace en 1964.

El Bocaina (Be8). Originalmente USS Marts (DE-174) de la armada estadounidense. Fue puesto en quilla el 26 de abril de 1943, lanzado el 08 de agosto de 1943 y comisionado el 03 de septiembre de 1943 en el astillero Federal Shipbuilding and Drydock Company de Newark, Nueva Jersey. $\mathrm{Su}$ primer comandante fue el teniente Carl M. Fellows. Descomisionado y transferido a la Marinha do Brasil el 20 de marzo de 1945 en donde se 
les denominó contratorpedeiro. Su primer comandante fue el capitán de corbeta Augusto Lopes da Cruz. El 30 de junio de 1953 pasó nuevamente al US Navy, solo para ser transferido formalmente a la Marinha do Brasil por el Programa de Asistencia Mutua de Defensa (MDAP). Su nombre fue retirado del Registro Naval de Buques de los Estados Unidos en 20 de julio de 1953. En 1955 se cambia su número de casco a D22. En 1960 fue reclasificado como Aviso Oceánico, retirándole todo el armamento antisubmarino y cambiando su número de casco a U32. Fue dado de baja y vendido para su desguace en 1975.

\section{LOS CANNON URUGUAYOS}

El Rou Uruguay (DE-1). Originalmente USS Baron (DE-166) de la armada estadounidense. Fue puesto en quilla el 30 de noviembre de 1942, lanzado el 09 de mayo de 1943 y comisionado el 05 de julio de 1943 en el astillero Federal Shipbuilding and Drydock Company de Newark, Nueva Jersey. Su primer comandante fue el teniente comandante D. McVicker del USNR. Fue descomisionado el 26 de abril de 1946. Su nombre fue retirado del Registro Naval de Buques de los Estados Unidos en 14 de mayo de1952. Transferido a la Armada Nacional de la República Oriental del Uruguay el 03 de mayo de 1952 por el Programa de Asistencia Mutua de Defensa (MDAP).Dado de baja en 1990. Fue hundido como blanco el 27 de febrero de 1995.

El Rou Artigas (DE-2). Originalmente USS Bronstein (DE-189) de la armada estadounidense. Fue puesto en quilla el 26 de agosto de 1943, lanzado el 14 de noviembre de 1943 y comisionado el 13 de diciembre de 1943 en el astillero Federal Shipbuilding and Drydock Company de Newark, Nueva Jersey. Su primer comandante fue el teniente Sheldon Hoard Kinney. Fue descomisionado el 05 de noviembre de 1945. Su nombre fue retirado del Registro Naval de Buques de los Estados Unidos en 14 de mayo de 1952. Transferido a la Armada Nacional de la República Oriental del Uruguay el 03 de mayo de 1952 por el Programa de Asistencia Mutua de Defensa (MDAP). Fue dado de baja y vendido para su desguace en 1988. 


\section{ESPECIFICACIONES TÉCNICAS DE LA CLASE CANNON}

Desplazamiento:

1,240 toneladas estándar

1,620 toneladas a plena carga.

Dimensiones:

-Eslora: 93.30 metros.

-Manga: 11.00 metros.

-Calado: 3.50 metros.

Maquinaria:

4 motores diesel General Motors GM 16-278A de 6,000 hp. 2 Hélices.

Velocidad:

-Máxima: 21 nudos.

Rango:

10,800 millas a 12 nudos.

Tripulación:

216; conformados por 15 oficiales y 201 suboficiales y marineros. Armamento:

-Artillería Naval

3 cañones Mk 22 de $76 \mathrm{~mm}$ y 50 calibres, en montajes simples.

2 cañones AB Bofors Mk1de 40 mm y 56 calibres, en un montaje doble.

8 cañones Oerlikon Mk4 de 20 mm en montajes dobles.

-Tubos lanzatorpedos

1 lanzador triple para torpedos de $533 \mathrm{~mm} \mathrm{Mk15.*}$

-Cohetes Antisubmarinos

1 lanzador Hedgehog Mk10 para cohetes antisubmarinos.

-Cargas de Profundidad

8 lanzadores K-gun para cargas de profundidad Mk6 o Mk9.

2 lanzaderas a popa para cargas de profundidad Mk3.

-Generador de Humo

2 generadores de humos Mk4. 
Electrónica:

1 radar de búsqueda en superficie SL.

1 radar de búsqueda aérea $\mathrm{SA}$.

2 sistemas de control de tiro Mk51 asociados al sistema Bofors Mk1.

Sonar:

1 sonar QCS-1.

*Durante su reacondicionamiento, previo a su incorporación a la Marina de Guerra del Perú les fue retirado el lanzador triple de torpedos. Por lo que los destructores de escolta peruanos no contaron con esta arma desde el inicio de su servicio en nuestra Marina.

\section{HISTORIAL DEL USS BANGUST (DE-739) EN LA ARMADA ESTADOUNIDENSE}

El USS Bangust (DE-739) fue un destructor de escolta de la clase Cannon construido para la armada de los Estados Unidos durante la Segunda Guerra Mundial. Sirvió en el Pacífico prestando servicio de escolta contra submarinos y ataques aéreos a los buques y convoyes de la armada. Regresó a Estados Unidos después de la guerra con un número casi record de once estrellas de batalla, solo superado por el USS Riddle ${ }_{3}$ (DE-185) con doce.

Esta unidad fue nombrada en honor de Joseph Bangust ${ }_{4}$ que fue galardonado póstumamente con la Cruz de la Marina por sus acciones contra los japoneses durante un combate aéreo a inicios de la guerra. El Bangust fue puesto en quilla el 11 de febrero de 1943, en los astilleros Western Pipe \& Steel Co de San Pedro, California. Fue botado el 6 de junio de 1943 amadrinado por la señora Stephen W. Gerber; finalmente fue comisionado el 30 de octubre de 1943, siendo su primer comandante el teniente comandante Charles F. MacNish del USNR.

El Bangust realizó su entrenamiento de alistamiento hasta el 21 de noviembre de 1943 llevando a cabo estas operaciones desde San Diego, California. Considerado listo para unirse a la flota después de la finalización de su entrenamiento el 18 de diciembre, el Bangust quedó a disponibilidad en la base naval de San Pedro, y finalmente se le informó a 
su comandante zarpar. El 9 de enero de 1944, dejó San Pedro para dirigirse a San Francisco, California, partiendo de allí el 13 de enero hacia Hawai, en compañía del Reynolds ${ }_{5}$ (DE-42). El Bangust llegó a Pearl Harbor el 19 de enero.

El Bangust fue el buque insignia de la División de Escolta (CortDiv) 32, al mando del comandante C.K. Hutchinson; zarpó en su primera misión de escolta el 25 de enero desde Pearl Harbor en compañía del Pastores (AF-16), el Ajax (AR-6) y el Wadleigh $_{8}$ (DD-689), todos con destino a las islas Ellice. La unidad de tarea se disolvió cuando llegó a Funafuti el 2 de febrero, luego continuó a las islas Gilbert ese mismo día, llegando a Makin el 5, trasladándose a Tarawa al día siguiente.

El 8 de febrero, navegó en compañía del Greiner $_{9}$ (DE-37) para reunirse con el Caliente ${ }_{10}(\mathrm{AO}-53)$ y tres mercantes para navegar a Majuro en las Islas Marshall. Sin embargo el Bangust y el Greiner recibieron nuevas órdenes, el 10, enviándolos de vuelta a Tarawa, donde llegaron ese mismo día. Después, el buque se puso en marcha nuevamente el 18, se reunió con el Fleming ${ }_{11}$ (DE-32) y el Anacapa ${ }_{12}$ (AG-49) para luego dirigirse a Kwajalein en las islas Marshall. El Bangust y las otras unidades llegaron a Roi Namur el 21, regresando los dos escoltas a Tarawa el 24.

El Bangust asistió luego al grupo de tarea 57.7 el 1 de marzo, en misión de escolta y patrullaje, operando en las inmediaciones de Tarawa hasta el 7. Entre el 8 y el 18, estuvo al ancla en Tarawa. Poco después, el 19, se unió al Eisele ${ }_{13}$ (DE-75) para escoltar al LST-29 ${ }_{14}$ a la laguna Abemama, llegando el mismo día.

Tres días más tarde, el Bangust, junto con el Eisele, navegaron hacia Pearl Harbor como escoltas del Kenmore ${ }_{15}$ (AK-221), del De Grasse ${ }_{16}$ (AK223) y del LST-29. Cumplida su misión, el mismo día, se le ordenó que volviera a Majuro. Al llegar, en la mañana del 24 de marzo, el destructor recibió la orden de ejecutar una misión antisubmarina del comandante del Escuadrón de Servicio 10. El destructor llegó al lugar de un ataque submarino anterior y llevó a cabo operaciones de guerra antisubmarina en la zona hasta volver a Majuro el día 27.

Dos días más tarde, el Bangust escoltó a los petroleros de la flota en una misión de abastecimiento en el marco de las operaciones de despliegue 
contra Hollandia, Nueva Guinea Holandesa, volviendo a Majuro el 5 de abril. Después de haber completado su misión, partió de las islas Marshall el 10 de abril, escoltando al Kaskaskia ${ }_{17}$ (AO-27) a mar abierto antes de volver a su base de operaciones.

De regreso a Majuro el 14 de abril, el Bangust pasó las siguientes semanas en una sucesión de misiones de escolta entre Majuro y Kwajalein, escoltando al LST-119 ${ }_{18}$ a Kwajalein entre el 18 y el 19 de abril. Al Capable ${ }_{19}$ (AM-155) y al ATR-44 ${ }_{20}$ a Majuro entre el 21 y el 23 de abril, destacándose un rendez vous con el Grouper ${ }_{21}$ (SS-214), para escoltar a este submarino a Majuro los días 26 y 27 de abril y operó en misión de patrulla en la entrada de este puerto entre el 29 y 30 de abril. El Bangust, junto con el Waterman (DE-740) y el Weaver (DE-741) zarparon luego el 3 de mayo escoltando al grupo de tarea 50.17 para volver el mismo día.

Aquí hacemos un alto en el desarrollo del historial de esta unidad, para hacer notar al lector que los tres buques que son motivo del presente capítulo, navegaron juntos, como acabamos de indicar, el 3 de mayo de 1944. Fue la primera y única vez que esto sucedió durante su servicio en el frente del Pacífico durante la Segunda Guerra Mundial. Nadie podía imaginar en aquel entonces que los tres buques navegarían juntos nuevamente; pero al ser incorporados a la Marina de Guerra del Perú ocho años más tarde.

Siguiendo con nuestro relato; el Bangust acompañó al Lackawanna ${ }_{22}$ (AO-40) a mar abierto del 4 al 6 de mayo, el destructor volvió entonces a Kwajalein el 8. Volviendo posteriormente a Majuro el 10 permaneciendo allí hasta que partió a Hawai el 16.

Al llegar a Pearl Harbor el 22 de mayo, el Bangust entró al astillero al día siguiente y se sometió a reparaciones hasta el 3 de junio. Entre los trabajos realizados se encontraban las incorporaciones del radar SL y reparaciones en la caldera auxiliar. Zarpó en solitario hacia las islas Marshall el 4 de junio. En un punto a unos 97 kilómetros de su destino, Roi Namur, el radar del Bangust tuvo un contacto a las 23:25 horas del 10 de junio de 1944. No habiendo recibido informes de ninguna nave en su derrota proyectada, el destructor rastreó el contacto continuamente hasta que los vigías divisaron un buque por proa hacia estribor a las 23:45 horas. La mala visibilidad dificultó la identificación; pero, como la distancia se 
acortó a solo 270 metros, los vigías del Bangust identificaron al objetivo probablemente como una embarcación pequeña o un submarino.

Después de que la señal de radar desapareciera a las 23:48 horas, el Bangust se detuvo en la zona del contacto y disparó una andanada de bengalas para iluminar el área, pero sin resultado. Apenas pasada la medianoche, inició una búsqueda por sonar, sin ninguna respuesta; entonces lanzó cuatro andanadas sucesivas de cohetes antisubmarinos, la última de las cuales provocó una serie de seis explosiones. Después de que cesaran, otra explosión más grande sacudió la nave.

Esa onda de choque hizo suponer a los marineros del destructor que habían sido torpedeados. Los equipos de control de daños comenzaron inmediatamente su tarea, pero sólo encontraron una pequeña vía de agua en la sala de máquinas delantera donde se iniciaron las reparaciones. A popa, los marineros revisaron los depósitos de las cargas de profundidad, pensando que un lanzador K-gun podría haber sido disparado accidentalmente, pero no encontraron cargas faltantes.

El operador de sonar del buque informó entonces que había oído dos explosiones más, pero bastante débiles, junto con ruidos silbantes y burbujeantes. Un fuerte olor a diesel se percibía en el aire cerca de la zona del ataque final. La contabilidad de la posguerra confirmaría que el Bangust había hundido al submarino japonés RO- $42_{23}$. El Bangust permaneció en la zona, continuando la búsqueda hasta las 17:00 horas del 11 de junio. El Greiner, acompañado de los dragaminas YMS-282 ${ }_{24}$ y YMS- $203_{25}$, así como del caza submarinos SC-1364 ${ }_{26}$ llegaron a la zona relevando al Bangust de las operaciones para poder reanudar su viaje a Roi Namur, al que llegó más tarde el mismo día.

Después de patrullar la entrada al puerto de Roi Namur, el 13 de junio, el Bangust se unió al Capps 27 (DD-550) y al Weaver para proteger un convoy con destino a Eniwetok. Al llegar a ese lugar el 15, el destructor se reabasteció y volvió a reunirse con el convoy dirigiéndose hacia las islas Marianas. Llegó a la zona de abastecimiento establecida al este de Saipán, el Bangust protegió a los petroleros mientras llevaban a cabo su servicio a la flota hasta el 20 de junio. En ese momento, se separó para escoltar a tres de los petroleros de vuelta a Eniwetok. Al llegar a su destino 
el 24, permaneció allí hasta el 29, cuando asumió la función de patrullar la entrada de este puerto.

Volvió a Eniwetok el 30 de junio, y permaneció allí hasta el 13 de julio, cuando se unió al Capps y al Weaver para proteger la salida del $\mathrm{Neosho}_{28}$ (AO-48) y del Lackawanna. El 15 de julio, se incorporó al grupo de tarea 50.17 y pronto operó en las inmediaciones de Guam y Saipán, protegiendo a los petroleros que mantenían a la "flota que llegó para quedarse" durante las operaciones de captura de Guam.

Cumplida esta misión el 23 de julio, el Bangust escoltó a sus buques de regreso a Eniwetok, llegando allí tres días después. Zarpó de nuevo hacia las islas Marianas, esta vez en compañía del Whitman ${ }_{29}(\mathrm{DE}-24)$, volviendo a unirse al grupo de tarea 50.17 en la zona de abastecimiento de Saipán el 2 de agosto. Un contacto sonoro el 3 de agosto obligó a separarse de los buques logísticos, mientras realizaba operaciones antisubmarinas con la esperanza de hundir a su segundo submarino enemigo. Una andanada de cinco cargas de profundidad disparadas a la zona de contacto no produjo ningún resultado, por lo que abandonó la misión y se reunió nuevamente con el convoy.

Al Bangust, al John D. Henley ${ }_{30}$ (DD-553) y al Fair ${ }_{31}$ (DE-35), el 11 de agosto se les ordenó fondear en Saipán. Posteriormente fue asignado para patrullar este puerto, el Bangust operó en esta misión el día 12. Después, al día siguiente, el buque salió en una breve misión de reconocimiento con la División de Escolta (CortDiv) 65. Al terminar esta misión, volvió a Saipán en compañía del Acree $_{32}$ (DE-167).

El Bangust protegió un convoy a Eniwetok sin incidentes entre el 16 y el 19 de agosto y, luego, disfrutó de un breve período de mantenimiento y disponibilidad del 20 al 25 de agosto en Eniwetok. Durante este tiempo, el comandante de la División de Escolta 32 cambió su insignia de comando del Bangust al Waterman el 23. El 26, el Bangust escoltó un convoy a Manus, islas del Almirantazgo, llegando allí el día 31. Allí se reabasteció y llevó a cabo reparaciones entre el 1 y el 9 de septiembre. Después de realizar misiones de escolta para otra unidad de petroleros, el buque estableció rumbo al puerto de Seeadler, llegando el 15 de septiembre. Tres días más tarde, el 18, el Bangust escoltó a tres unidades a una misión de 
abastecimiento para los buques de la $3^{\mathrm{a}}$ flota estadounidense, uniéndose al grupo de tarea 38.3 el 26 de septiembre. Luego fue asignado al grupo de tarea 30.8, y el buque escoltó a la unidad de tarea de regreso a las islas del Almirantazgo, llegando a Manus el $1^{\circ}$ de octubre.

El Bangust se sometió a reparaciones y preparativos para sus próximas operaciones hasta el 9 de octubre y luego navegó en compañía del $\mathrm{Mascoma}_{33}(\mathrm{AO}-83)$ el día 10, con destino al estrecho de Kossol. Al llegar a su destino el 13 de octubre, permaneció allí hasta el 18, cuando se puso en marcha en compañía del Niobrara $_{34}(\mathrm{AO}-72)$, con destino a las islas del Almirantazgo. Al llegar a Manus el 21 de octubre, el destructor navegó en compañía del Swearer ${ }_{35}$ (DE-186) el día 25, escoltando una unidad de tarea con destino a las islas Carolinas occidentales. Los buques llegaron a Ulithi el 28 de octubre sin incidentes. El Bangust permaneció en Ulithi durante los siguientes días, realizando varias misiones de patrulla antes de su partida el 12 de noviembre como escolta de un grupo de petroleros que se dirigía a un encuentro con la flota.

Después de esta misión, el Bangust escoltó al portaaviones Nehenta $\mathrm{Bay}_{36}(\mathrm{CVE}-74)$, antes de dirigirse a realizar otras misiones antes de recibir órdenes de volver a Ulithi el 22 de noviembre. Llegando a su destino el 24, el buque volvió a ser el buque insignia de la División de Escolta 32, el 28 de noviembre. El 10 de diciembre salió con el grupo de tarea 30.8, volviendo a Ulithi el 11 para reparaciones.

El 12 de diciembre de 1944, el Bangust comenzó una misión de escolta con la 3ra flota. Entre el 18 y 19 de diciembre el tifón Cobra dañó varios buques y hundió tres destructores. Durante la noche del 18, el Bangust estuvo al lado del destructor Aylwin 37 (DD-355) participando en los esfuerzos de búsqueda de sobrevivientes de dos de los tres destructores hundidos; el SPENCE 38 (DD-512) y el Hull ${ }_{39}$ (DD- 350).

Culminada la búsqueda el día 23, volvió a Ulithi llegando a su destino el día de Navidad de 1944. Dos días más tarde, el comandante de la División de Escolta 32 cambió su insignia al Kyne ${ }_{40}$ (DE-744). El Bangust zarpó de Ulithi el 3 de enero de 1945 como escolta de otro grupo de reabastecimiento de combustible, pero retornó después de sólo un día, para efectuar reparaciones, encontrándose con el Chikaskia ${ }_{41}(\mathrm{AO}-54)$ al cual escoltó hasta Ulithi, llegando el 5. El 7, el Bangust zarpó al golfo de Leyte, llegando el 
día 14, y al mar de Sulu al día siguiente, antes de que finalmente se uniera al grupo de tarea 30.8 el día 16 en el mar de China Meridional. Procediendo de allí a Ulithi vía el golfo de Leyte. El Bangust llegó a su destino el 18. Desde aquí escoltó al Neches ${ }_{42}$ (AO-47) a San Pedro Bay, Leyte.

El Bangust se puso en marcha para una misión de abastecimiento el 21 de enero, en compañía del Neches, y se unió a otros petroleros de la flota y escoltas durante la travesía. Se separó junto con el Tomahawk ${ }_{43}(\mathrm{AO}-$ 88), el Merrimack ${ }_{44}(\mathrm{AO}-37)$ y el Crowley ${ }_{45}(\mathrm{DE}-303)$ y le fue ordenado proceder a las islas Carolinas occidentales, llegando a Ulithi el 25 de enero. Cuatro días después, el comandante de la División de Escolta 32 volvió a colocar su insignia en el Bangust.

Después de completar reparaciones, navegó junto al Neches el 8 de febrero de 1945 y los petroleros del grupo de tarea 50.8, y con esta unidad abastecieron a los buques de los grupos de tarea $58.1,58.4$ y 58.5 el 19 de febrero y a al 58.2 y 58.3 el 20. Al terminar esta misión, el día 21, el Bangust escoltó a una unidad de tareas de regreso a las islas Carolinas occidentales, llegando a Ulithi el 23, donde inmediatamente se unió a otra unidad de tareas, y finalmente regresó al grupo de tarea 50.8 el 26 de febrero, volviendo a Ulithi el 5 de marzo.

El Bangust regresó a la zona de avanzada el 25 de marzo, escoltando a los petroleros a su encuentro con el grupo de tareas de los portaviones. El Bangust rastreó en y alrededor de las áreas de abastecimiento de combustible buscando submarinos hasta retirarse el 5 de abril, llegando a Ulithi el 9.

El Bangust fue sometido a reparaciones en esa base hasta el 16, cuando reasumió sus operaciones con los petroleros que abastecían los buques que participaban de las operaciones en Okinawa. Uniéndose al grupo de tarea 50.8 el 19 de abril, el Bangust operó en misiones antisubmarinas y antiaéreas. El 7 de mayo zarpó hacia Guam llegando a Apra el día 10 y se puso en marcha el 20 de mayo para reunirse con grupo de tarea 50.8. El 24 de mayo el grupo de tarea fue renombrado 30.8 en misión de escolta a petroleros en una operación de abastecimiento.

El Bangust permaneció en alta mar con estos buques hasta el 7 de junio, cuando se le ordenó regresar a Guam. Llegando a Apra el 10, el 
buque fue sometido a reparaciones menores y mantenimiento, reabasteció municiones y provisiones mientras su tripulación disfrutó de un período de descanso. El 23 de junio, el Bangust realizó un corto viaje de Guam a Saipán y permaneció allí, aguardando órdenes, hasta el 3 de julio de 1945.

El 4 de julio, el Bangust zarpó en compañía de los portaaviones Admiralty Islands ${ }_{46}$ (CVE-99), Hollandia ${ }_{47}$ (CVE-97), Thetis Bay ${ }_{48}$ (CVE90), Roi ${ }_{49}(\mathrm{CVE}-103)$ y los destructores Reynolds (DE-42), McClelland ${ }_{50}$ $(\mathrm{DE}-750)$ y Thorn $_{51}$ (DD-647), para encontrarse con el grupo de tarea 30.8; a este grupo de tareas se le asignó el trabajo del apoyo logístico a los portaaviones de la fuerza de tarea 38 durante los ataques a Tokio y otros objetivos al norte de Japón. Después de cumplir su misión el 5 de julio, el Bangust operó con el grupo de tarea 30.8 hasta que el 18 zarpó a Ulithi a donde llegó el 23. El 25, el Bangust realizó un patrullaje antisubmarino para cubrir la salida de buques de Ulithi y luego se dirigió a unirse al grupo de tarea 30.8 el 29.

Tras la capitulación de Japón a mediados de agosto, el Bangust se mantuvo en alta mar con el grupo de tarea 30.8. El 27 de agosto, después de haber embarcado un equipo de asalto, se dirigió a un punto de encuentro para hacer efectiva la rendición del submarino japonés I- $14_{52}$. Acompañado por cuatro aviones de combate Grumman F4F Wildcat y el Murray $_{53}$ (DD-576), el Bangust realizó con éxito su misión el 28 y navegó con el submarino japonés I-14 y el Proteus ${ }_{54}$ (AS-19), a Sagami Wan para desembarcar a la tripulación del submarino japonés rendido; El 30, el destructor fue abastecido por el Mascoma (AO-83). Luego zarpó hacia la bahía de Tokio el 31, llegando allí por la tarde del mismo día. Tras una breve pausa, se dirigió al día siguiente hacia Saipán, llegando a su destino el 5 de septiembre. Dos días después, el Bangust, en compañía del Kyne, volvió a Japón con un convoy de cuatro mercantes. Al llegar a Tokio el 13, el destructor se desplazó a Yokosuka Ko, al día siguiente.

El Bangust zarpó hacia los Estados Unidos el 2 de octubre de 1945 en compañía de otros buques de su división y, después de una breve escala en Pearl Harbor entre el 10 y el 13, llegó a San Pedro, el 20. Estuvo durante dos semanas en mantenimiento y salió el 6 de noviembre hacia el Canal de Panamá llegando el 16 y procediendo a cruzarlo saliendo de aguas panameñas el 17. El destructor llegó al astillero naval de Filadelfia, 
Pensilvania el 22 de noviembre. Luego pasó a Green Cove Springs, Florida, donde después de pasar revisión fue desarmado el 14 de junio de 1946. Finalmente fue descomisionado el 17 de noviembre de 1946. El 21 de marzo de 1947, el Bangust fue trasladado a la base naval de Charleston, Carolina del Sur, remolcado por el Challenge ${ }_{55}$ (ATA-201) entre el 17 y 18 de junio. Permaneció allí hasta el 13 de agosto, cuando remolcado por el Tunica ${ }_{56}$ (ATA-178) fue llevado nuevamente a Green Cove Springs, llegando al día siguiente.

El Bangust fue transferido a la Marina de Guerra del Perú el 26 de octubre de 1951 bajo los términos del Programa de Asistencia de Defensa Mutua (MDAP). Su nombre fue retirado del Registro de Naval de Buques el 18 de abril de 1952. Fue renombrado B.A.P. Castilla (D-1).

\section{HISTORIAL DEL USS WATERMAN (DE-740) EN LA ARMADA ESTADOUNIDENSE}

El USS Waterman (DE-740) fue un destructor de escolta de la clase Cannon construido para la armada de los Estados Unidos durante la Segunda Guerra Mundial. Sirvió en el Pacífico y prestó servicio de escolta contra submarinos y ataques aéreos a los buques y convoyes de la armada. Regresó a Estados Unidos después de la guerra con ocho estrellas de batalla, mucho más que el promedio de los destructores de escolta.

Esta unidad fue nombrada en honor de Andrew Kenneth Waterman ${ }_{55}$, que fue galardonado póstumamente con la Cruz de la Marina por sus acciones contra los japoneses durante un combate aéreo a inicios de la guerra. El Waterman fue puesto en quilla el 24 de febrero de 1943, en los astilleros Western Pipe \& Steel Co de San Pedro. Fue botado el 20 de junio de 1943, amadrinado por la Sra. June M. Waterman, viuda de Andrew Kenneth Waterman. Finalmente fue comisionado el 30 de noviembre de 1943; su primer comandante fue el teniente comandante W. B. Hinds, USNR.

Después de realizar su entrenamiento de alistamiento el Waterman zarpó de San Pedro, el 12 de febrero de 1944 y navegó en solitario a Pearl Harbor, donde llegó el 18 de febrero. Una vez en aguas hawaianas, el buque recibió entrenamiento adicional en lucha contra submarinos y de artillería. 
El Waterman salió de Pearl Harbor el 6 de marzo escoltando al portaaviones $\mathrm{Nassau}_{56}(\mathrm{CVE}-16)$ que transportaba aviones, tropas y carga a Kwajalein, Majuro, Tarawa y Makin en las islas Marshall y las islas Gilbert. El Waterman volvió a Pearl Harbor el 24 de marzo.

Continuando en su misión de escolta, el Waterman zarpó desde Hawai el 9 de abril, hacia las islas Marshall en compañía del Bowers ${ }_{57}$ (DE637) y escoltó al convoy 4152-A, compuesto de buques mercantes llegando a Majuro una semana más tarde, para luego realizar misiones locales de escolta desde esa base hasta mayo.

El Waterman, junto con el Bangust y el Weaver zarparon de Majuro el 3 de mayo escoltando al grupo de tarea 50.17 volviendo el mismo día. El $1^{\circ}$ de junio, el Waterman se volvió a unir al grupo de tareas 50.17, un grupo compuesto de buques de apoyo; en particular, petroleros, remolcadores, municionadores y similares; que permitían a la flota de mar operar durante largos periodos de tiempo. Esos buques proporcionaban a los portaaviones y a sus escoltas requerimientos básicos como alimentos, combustible, municiones, etc. todo lo que la flota necesitaba para mantener la presión sobre los japoneses.

La primera asignación del Waterman en misión de apoyo fue en la operación de las islas Marianas. Salió de Majuro el 6 de junio y protegió al grupo de tareas durante quince días antes de completar su misión en Eniwetok el día 20. Luego se dirigió a las islas Marianas y escoltó a un petrolero desde Saipán, mientras aún se combatía en tierra; retornando a las islas Marshall.

De nuevo en Eniwetok el 26 de julio, el Waterman se reunió con el grupo de servicio de la flota al este de las islas Marianas y protegió a los petroleros mientras reabastecían a los buques que apoyaban los desembarcos en Guam.

El 23 de agosto el comandante de la División de Escolta 32 cambió su insignia de comando al Waterman desde el Bangust. Después de regresar a las islas Marshall, el Waterman zarpó de Eniwetok el 26 de agosto, con destino a las islas del Almirantazgo y llegó a Manus cinco días después. Poco después, regresó a mar abierto con una unidad de la Fuerza de Servicio 30.8.7, apoyando la invasión de las islas Carolinas occidentales. 
Durante ese tiempo, el destructor operó al oeste de las Filipinas, soportando y repeliendo ataques aéreos japoneses dirigidos principalmente contra los portaaviones. El Waterman operó desde Manus hasta el 4 de octubre y luego fue destacado a escoltar al grupo de servicio de la flota al este de Filipinas, donde se reabastecían a los portaaviones que lanzaban ataques aéreos contra las posiciones japonesas en la isla de Leyte.

Después de escoltar a las unidades de la fuerza que estaban apoyando los desembarcos en Leyte, el Waterman operó entre el 2 de noviembre y el 23 de diciembre con el grupo de tarea 30.8; el grupo de tareas que prestaba servicio a las fuerzas que operaban al este de las Filipinas; Los aviones de estas unidades neutralizaron en gran medida el poder aéreo y marítimo japonés en Filipinas y Formosa. El 24 de noviembre, el buque dejó de ser el buque insignia de la División de Escolta (CortDiv) 32, pasando la insignia nuevamente al Bangust.

Mientras operaba con el grupo de tarea 30.8, el Waterman encontró el peor clima de su carrera, el tifón Cobra, el 18 de diciembre de 1944. Durante aproximadamente 36 horas, la feroz tormenta golpeó a la flota del almirante William F. Halsey, afectando a buques grandes y pequeños por igual. Los vientos de 220 kilómetros por hora y las olas de unos 24 metros de altura hicieron que la vida a bordo del Waterman fuera muy difícil. En ocasiones, el barco escoró hasta $65^{\circ}$. Como recordara su comandante:

\section{"Este día fue un día inolvidable y está indeleblemente impreso en la mente de la tripulación"}

Antes de que el tifón hubiera perdido su fuerza, tres buques; todos destructores, se habían hundido y otros buques resultaron dañados. El 23 de diciembre, el Waterman regresó a Ulithi algo maltratado, pero en mejor condición que la mayoría de los buques más grandes.

Una semana después, el destructor volvió a estar a la mar, rumbo a Guam. Desde el 4 de enero de 1945 hasta el 3 de marzo, el Waterman estuvo en servicio continuo de protección del grupo de servicio de la flota. Pasó gran parte de enero apoyando la ocupación de Luzón desde las zonas de abastecimiento al este de Filipinas y en febrero escoltó a los buques auxiliares a encontrase con la flota que pronto comenzó el bombardeo de Iwo Jima; antes de la invasión. 
Mientras se encontraba en misión, el Waterman se distinguió el 17 de febrero; una explosión interna destruyó la sección de proa del petrolero Patuxent ${ }_{58}$ (AO-44), dejando aberturas en su casco y fuego que hicieron estragos sobre la proa del barco poniendo en peligro la carga de combustible. El Waterman rápidamente se dirigió; y fue el primero, de los dos escoltas que se acercaron a ayudar al petrolero. Se aproximó al buque en peligro de un lado, mientras que el Dewey ${ }_{59}$ (DD-349) se acercaba desde el otro lado. Los equipos de extinción de fuegos de los destructores, operando en condiciones extremadamente peligrosas lanzaron miles de litros de agua sobre el fuego del Patuxent logrando extinguirlo. Por dirigir el esfuerzo de los destructores de escolta, el teniente comandante. J. H. Stahle, USNR, comandante del Waterman, recibió la Estrella de Bronce.

Después de pasar por mantenimiento y logística en Ulithi, el Waterman partió de las islas Carolinas occidentales el 22 de marzo hacia una zona de abastecimiento al este de Okinawa. Durante las primeras dos semanas de abril, el Waterman escoltó al portaaviones ATTU ${ }_{60}$ (CVE-102) mientras transportaba aviones de reemplazo para las fuerzas de tarea en dos viajes de ida y vuelta entre Okinawa y Guam.

Durante el resto de la guerra en el Pacífico, el Waterman escoltó a las unidades de servicio de la flota que operaban a unos cientos de kilómetros de Japón, mientras que los portaaviones, los acorazados y los cruceros llevaban a cabo ataques devastadores en territorio enemigo.

El 21 de agosto, menos de una semana después de la capitulación de Japón, el Waterman fue asignado al grupo de tarea 35.80, un grupo especial de apoyo establecido para entrar en la bahía de Tokio como parte de la fuerza de ocupación inicial. Con su bandera a tope, el Waterman y el Kyne; fueron los primeros destructores de escolta en llegar a Sagami Wan; entraron en la bahía justo al suroeste de la capital japonesa, el 28 de agosto y echaron anclas a menos de una milla de la costa de la ciudad de Katase.

El31 de agosto, el Waterman se trasladó a la bahía de Tokio propiamente dicha y, dos días más tarde, izó su bandera cuando se firmó la rendición de Japón a bordo del acorazado Missouri ${ }_{61}$ (BB-63). El 4 de septiembre, 
el Waterman fue asignado al grupo de tarea 30.6, cuyo deber era evacuar prisioneros de guerra aliados de los campos de prisioneros cercanos. Esa tarde, el destructor entró en el puerto de Yokohama y transportó prisioneros de guerra a los buques hospital. El Waterman continuó su trabajo hasta el 10 de septiembre, cuando partió del área de Tokio con el grupo de tarea 30.6, compuesto por cuatro LSM y el Weaver con destino a Shiogama, en la costa oriental de Honshu, ya que a través de este puerto los prisioneros del campamento de Sendai estaban siendo evacuados.

A la mañana siguiente, el Waterman entró en el puerto de Shiogama y se unió a otras unidades del grupo de tarea 30.6 que ya estaban en el proceso de evacuación de los prisioneros. El 14 de septiembre, el destructor zarpó hacia Kamaishi, llegando allí a la mañana siguiente para la evacuación posterior de prisioneros.

Una vez completada dicha operación, el grupo de tarea 30.6 regresó a la bahía de Tokio donde se disolvió; el Waterman fue asignado para tareas de escolta con el grupo de tarea 16.5 del escuadrón de servicio 6. En esta misión, el destructor permaneció en el puerto de Yokosuka del 19 al 29 de septiembre.

En la tarde del 29 de septiembre, el Waterman recibió órdenes de regresar a Estados Unidos después de 20 meses de servicio en la zona de guerra del Pacífico; partiendo de la bahía de Tokio el 2 de octubre.

Después de navegar vía Pearl Harbor, llegó a San Pedro, el 20 de octubre y permaneció allí hasta el 6 de noviembre, cuando se puso en marcha hacia el Canal de Panamá y de ahí a Filadelfia llegando el 22 de noviembre, el buque permaneció en Filadelfia hasta el 10 de diciembre, en disponibilidad. Poco después, el Waterman se trasladó a Green Cove Springs, donde fue descomisionado el 31 de mayo de 1946.

El Waterman fue transferido a la Marina de Guerra del Perú el 26 de octubre de 1951 bajo los términos del Programa de Asistencia Mutua de Defensa (MDAP). Su nombre fue retirado del Registro de Naval de Buques el 18 de abril de 1952. Fue renombrado B.A.P. Aguirre (D-2). 


\section{HISTORIAL DEL USS WEAVER (DE-741) EN LAARMADA ESTADOUNIDENSE}

El USS Weaver (DE-741) fue un destructor de escolta de la clase Cannon construido para la armada de los Estados Unidos durante la Segunda Guerra Mundial. Sirvió en el Pacífico y prestó servicio de escolta contra submarinos y ataques aéreos a los buques y convoyes de la armada. Regresó a Estados Unidos después de la guerra con nueve estrellas de batalla, mucho más que el promedio de los destructores de escolta.

Esta unidad fue nombrada en honor de Luther Dayton Weaver ${ }_{62}$ que murió durante el ataque japonés a Pearl Harbor el 7 de diciembre de 1941. El Weaver fue puesto en quilla el 13 de marzo de 1943, en los astilleros Western Pipe \& Steel Co de San Pedro, California. Fue botado el 4 de julio de 1943, amadrinado por la señora John Franklin Weaver, madre de Luther Dayton Weaver. Finalmente fue comisionado el 31 de diciembre de 1943; su primer comandante fue el teniente comandante R. S. Paret, USNR.

El Weaver llevó a cabo su entrenamiento de alistamiento a lo largo de la costa de California durante enero y febrero de 1944. El 2 de marzo, salió de San Francisco con destino al frente del Pacífico. El Weaver hizo una escala en Pearl Harbor entre el 14 y el 15 de marzo y después continuó su viaje a las islas Marshall vía Kwajalein. Llegó a Majuro a fines de ese mes y se unió al grupo de tarea 50.17; el grupo de reabastecimiento de combustible de la flota. El Weaver operó como una unidad de escolta de la 3ra flota durante su servicio en la II Guerra Mundial.

El 13 de junio de 1944, el Weaver, el Bangust y el Capps se unieron para proteger un convoy con destino a Eniwetok. El 13 de julio el Weaver se unió al Bangust y al Capps para proteger la salida de los petroleros Neosho y del Lackawanna desde Eniwetok.

Operando desde su base en Majuro, escoltó a los petroleros para encontrarse con los portaaviones durante las incursiones en Truk, Satawan y Ponape a fines de abril y principios de mayo. Avanzando a la base de Eniwetok, poco después, siguió protegiendo al grupo de logística durante el asalto a Saipán en junio. Más tarde mantuvieron a los portaaviones en acción durante la invasión de las islas Carolinas occidentales y las islas Palaos. Después de esa operación, el grupo logístico avanzó otra vez 
operando brevemente fuera del puerto de Seeadler en Manus y fuera de Ulithi por el resto de la guerra.

Ulithi sirvió de base para el grupo de tarea 58.38 durante el último año de la guerra en el Pacífico. El Weaver escoltó a los petroleros a Ulithi, donde volvieron a cargarse de petróleo y luego zarparon para abastecer a los portaaviones. Así, en 1945, ayudó a mantener la presión sobre los japoneses durante los desembarcos en Luzón, el asalto de Iwo Jima y durante la campaña de Okinawa. Las últimas fases de su servicio también incluyeron misiones de escolta en apoyo de las incursiones a Japón durante el verano de 1945.

Cuando los japoneses capitularon, el 15 de agosto de 1945, el Weaver estaba en alta mar con el grupo de tarea 30.8 protegiendo a los petroleros. El 28 de agosto, llevó un equipo de asalto desde el USS Proteus al submarino japonés rendido I $-400_{63}$ y luego entró en Sagami Wan, para comenzar su servicio con las fuerzas de ocupación. Durante el mes siguiente, el destructor ayudó en la evacuación de prisioneros de guerra aliados de Japón.

El 10 de septiembre, el Weaver partió del área de Tokio con el grupo de tarea 30.6, compuesto por cuatro LSM y el Waterman con destino a Shiogama, en la costa oriental de Honshu, porque por este puerto estaban siendo evacuados los prisioneros de guerra del campamento de Sendai.

El 2 de octubre terminó su misión en Japón y zarpó de Yokosuka con destino a Estados Unidos. La travesía fue vía Pearl Harbor, San Pedro, y el Canal de Panamá, para pasar finalmente a Filadelfia, llegando el 22 de noviembre para comenzar los preparativos para su inactivación. A fines de diciembre, se trasladó a Green Cove Springs, donde, aunque técnicamente todavía se encontraba comisionado, se unió a la Flota de Reserva del Atlántico. El Weaver finalmente fue descomisionado el 03 de abril de 1947.

El Weaver fue transferido a la Marina de Guerra del Perú el 26 de octubre de 1951 bajo los términos del Programa de Asistencia Mutua de Defensa (MDAP). Su nombre fue retirado del Registro de Naval de Buques el 18 de abril de 1952. Fue renombrado B.A.P. Rodríguez (D-3). 


\section{INCORPORACIÓN DE LOS DESTRUCTORES CANNON A LA MARINA DE GUERRA DEL PERÚ}

Durante el gobierno del Presidente Manuel A. Odría la Marina de Guerra del Perú impulsa un programa de modernización de nuestro poder naval adquiriendo unidades de combate de mediano tonelaje, puesto que el Ministro de Marina de ese entonces, contralmirante Roque Saldías Maninat estaba convencido de que aquel era el tipo de buque que más convenía a nuestra marina. Para Saldías, el destructor era la unidad de superficie idónea, tanto por aspectos económicos derivados del mantenimiento, gasto de combustible y de sostenimiento en el mar, así como por su flexibilidad para llevar a cabo operaciones independientes en cualquier área del dominio marítimo nacional.

El alto mando naval se esforzó por obtener la cesión o venta de unidades modernas provenientes de los excedentes de guerra de la armada estadounidense, pero razones de política exterior y nuestros limitados recursos, hacían difícil toda adquisición. La Marina de Guerra del Perú trabajó intensamente para obtener la dación de leyes especiales que otorgaran los fondos para este objetivo.

Es así que en el mes de octubre nos fueron transferidos por el gobierno de los Estados Unidos los tres destructores de escolta de la clase Cannon, veteranos de la II Guerra Mundial, que habían combatido en el Pacífico como lo hemos descrito líneas arriba; por un valor total de $\mathrm{S} /$. 62’098,227.02, que al tipo de cambio de la época, ascendía a un total de USD $\$ 40^{\prime} 181,698.80$, por las tres unidades de segundo uso, pero con menos de diez años de haber sido puestas en servicio en la armada estadounidense.

Las tres naves; el USS Bangust (DE-739), el USS Waterman (DE-740) y el USS Weaver (DE-741) fueron transferidos oficialmente a la Marina de Guerra del Perú el 26 de octubre de 1951 a través del Programa de Ayuda de Defensa Mutua (MDAP). Las tres unidades fueron denominadas por nuestra Marina de Guerra como BAP Castilla (D-1), BAP Aguirre (D-2) y BAP Rodríguez (D-3) respectivamente. Los nombres corresponden en el caso del primer buque, al Gran Mariscal Don Ramón Castilla, Presidente del Perú en el siglo XIX y gran impulsor del poder naval del Perú. El segundo y el tercero, por el comandante Elías Aguirre y por el teniente primero Melitón Rodríguez; segundo y cuarto comandante respectivamente 
del monitor Huáscar durante el combate naval de Angamos, muertos heroicamente en dicha gesta.

Inmediatamente, a fines de mes las tripulaciones peruanas, compuestas por 480 hombres y 42 oficiales llegaron a Green Cove Springs, Florida, base de la Flota de Reserva del Atlántico, ayudando a oficiales y hombres del US Navy en la reactivación y reacondicionamiento de los buques, trabajos que fueron realizados entre Green Cove Springs y Jacksonville.

Antes de su llegada, las tripulaciones de los nuevos buques habían recibido un entrenamiento técnico intensivo en las escuelas de la armada estadounidense para adaptarlas a las características de los nuevos destructores. Cabe destacar que al llegar por primera vez a la estación naval de Green Cove Springs, los suboficiales y marineros fueron acomodados en un buque atracado en la base y los oficiales, en los cuartos de oficiales que estaban de licencia. Sin embargo, tan pronto como el trabajo de acondicionamiento en los destructores estuvo avanzado, las tripulaciones se trasladaron a sus respectivas unidades.

Aquí un detalle muy importante; en el proceso de reacondicionamiento y activación de los destructores les fueron retirados el lanzador triple de

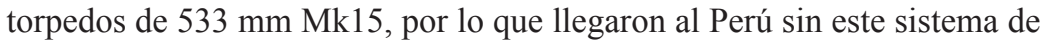
armas.

Hay que hacer referencia también a un hecho curioso; durante la estancia de nuestras tripulaciones en Florida, en un espléndido gesto de buena voluntad, aproximadamente la mitad de nuestros hombres se ofrecieron voluntariamente a donar sangre para el Programa de Donantes de Sangre de las Fuerzas Armadas estadounidenses, cubriendo así toda la cuota de la Flota de Reserva del Atlántico de un mes.

La ceremonia de incorporación y afirmado del pabellón nacional de las naves se realizó en la Estación Naval de Green Cove Springs, el jueves 21 de febrero de 1952 y contó con la presencia de un gran número de funcionarios civiles, militares y navales del área de Jacksonville.

Los buques fueron recibidos en representación del gobierno peruano por nuestro embajador en los Estados Unidos Sr. Fernando Berckemeyer Pazos acompañado de nuestro agregado naval en este país capitán de 
fragata Luis Castro Reus. Los nuevos destructores formarían la División de Destructores; al mando del capitán de navío Luis Edgardo Llosa Gonzales Pavón; sus primeros comandantes fueron; los capitanes de fragata Juan Fernando Lino Zamudio en el Castilla; Enrique León de la Fuente en el Aguirre y Enrique Carbonel Crespo en el Rodríguez.

Los buques fueron formalmente transferidos por el capitán Cauncey Moore USN, Comandante de la Flota de Reserva del Atlántico, que luego de firmar los certificados de transferencia, le fueron entregados a nuestro embajador, que a su vez se lo hizo al capitán Llosa, con lo que las naves fueron incorporadas a la Marina de Guerra del Perú. El capitán Llosa explicó brevemente los nombres de las nuevas unidades para posteriormente, en compañía del comandante H.P. McNally USN, capellán mayor de la Estación Naval de Green Cove Spring y del resto de los oficiales proceder a bendecir las unidades. Luego de bendecir las naves, el capitán Llosa ordenó a los comandantes de los destructores afirmar el pabellón nacional, a la vez que la banda de músicos de la Estación Naval tocó nuestro Himno Nacional, terminado el izado de nuestra bandera, las tripulaciones lanzaron tres veces el glorioso ¡Viva el Perú!

\section{HISTORIAL DE LOS DESTRUCTORES CANNON EN LA MARINA DE GUERRA DEL PERÚ}

El 24 de mayo de 1952 los destructores llegan a aguas peruanas y se encuentran con la comitiva de recepción compuesta por los submarinos $\mathrm{R}-1_{64}, \mathrm{R}-2_{65}, \mathrm{R}-3_{66}, \mathrm{R}-4_{67}$ y las fragatas Teniente Palacios ${ }_{68}$ y Teniente Ferré ${ }_{69}$ frente a Chancay; además se incorpora también a esta formación la fragata Teniente Gálvez $z_{70}$ que regresaba de una modernización en Estados Unidos. Todas las naves pasaron a Ancón, donde los destructores fueron inspeccionados por el Presidente de la República Manuel A. Odría llegando al Callao al día siguiente.

El 19 de julio de 1954 los destructores junto con los submarinos R-1, R-2, R-3 y R-4 forman la comitiva de recepción de los nuevos submarinos Tiburón $_{71}$ y Lobo ${ }_{72}$ en Ancón donde fueron inspeccionados por el Presidente de la República Manuel A. Odría; que embarcó en el Tiburón, partiendo la comitiva al Callao a donde llegaron en la tarde del miércoles 21 de julio. 
En 1955 los destructores participan del crucero de verano por varios puertos del litoral con los submarinos R-1, R-2, R-3 y R-4 Durante el mismo se les unen los submarinos Tiburón y Lobo para realizar entrenamientos, el grupo estuvo al mando del capitán de navío Juan Francisco Torres Matos.

En 1956 los destructores participan de un período de entrenamiento en San Diego con los submarinos R-1, R-2, R-3 y R-4. El grupo estuvo al mando del capitán de navío Julio Giannotti Landa.

A fines de 1958 los destructores participan de un período de entrenamiento en San Diego, retornando al Perú el 7 de diciembre.

Del 5 al 16 de marzo de 1959 los destructores llegaron a Estados Unidos para participar en los ejercicios antisubmarinos peruano-estadounidenses. Las operaciones incluyeron ejercicios de artillería, navegación, operaciones en el centro de combate y comunicaciones. A fines de este año los destructores, pasan a denominarse destructores de escolta y cambian de número de casco a Castilla (DE-1), Aguirre (DE-2) y Rodríguez (DE-3).

En 1960 los destructores de escolta realizan el crucero de verano con el nuevo crucero Almirante $\mathrm{Grau}_{73}$ y 237 cadetes visitando Paita, Pimentel, Salaverry, Chimbote, Ilo, Mollendo, Matarani y Pisco retornando el 25 de marzo.

El 5 de octubre se realiza una Revista Naval en conmemoración del $81^{\circ}$ aniversario del Combate naval de Angamos que contó con la asistencia del Presidente de la República Manuel Prado Ugarteche, así como de altas autoridades navales, militares y civiles. En la revista participaron los destructores de escolta junto con los cruceros Almirante Grau y Coronel Bolognesi $i_{74}$, así como los submarinos Dos de $\mathrm{Mayo}_{72}, \mathrm{Abtao}_{71}$, Iquique $_{75}$ y Angamos ${ }_{76}$. Los destructores de escolta lanzaron cargas de profundidad y cohetes antisubmarinos, luego de haber efectuado diversas evoluciones tácticas. Nuevamente a fines de este año los destructores de escolta cambian de número de casco a Castilla (DE-61), Aguirre (DE-62) y Rodríguez (DE-63).

En 1964, entre los meses de marzo y abril los destructores de escolta realizaron ejercicios de entrenamiento entre el Callao y Huacho, 
que comprendieron ejercicios de tiro en el polígono de Salinas, guerra antisubmarina, transferencia y otros. Luego los ejercicios continuaron entre el $1^{\circ}$ de agosto y el 24 de septiembre en el área entre el Callao y Pisco.

Entre el 21 de octubre y el 3 de noviembre se desarrolló la operación UNITAS V en la que participaron el Castilla y el Aguirre, con el crucero Almirante Grau, los destructores Villar 77 y Guise $_{78}$, las corbetas Gálvez y Diez $\mathrm{Canseco}_{80}$, el petrolero Talara ${ }_{81}$ y el submarino Iquique formando la Fuerza de Tarea 11. Por la armada estadounidense participaron los destructores Norfolk ${ }_{82}$, y John Willis $_{83}$, además del submarino Odax ${ }_{84}$, junto con dos aviones Lockheed P-2V Neptune y un Lockheed C-130 Hércules.

En diciembre el Aguirre y los cruceros Almirante Grau y Coronel Bolognesi junto con los destructores Villar y Guise realizaron ejercicios de tiro antiaéreo en el área de operaciones entre Jaguay y punta Sacasemita, al sur de Lima, con la colaboración de un bombardero ligero Douglas A-26 Invader de la Fuerza Aérea del Perú que remolcaba la manga con el blanco.

En 1966 entre el 18 y el 28 junio el Aguirre junto del crucero Coronel Bolognesi, el destructor Guise, la corbeta Gálvez, las cañoneras torpederas Velarde 85 , Santillana 8 , De Los Heros $_{87}$, Herrera $_{88}$, Larrea $_{89}$ y Sánchez Carrión ${ }_{90}$, así como con los submarinos Dos de Mayo e Iquique participa en ejercicios conjuntos con los submarinos argentinos Santa $\mathrm{Fé}_{91}$ y Santiago Del Estero ${ }_{92}$ que retornaban de San Francisco en donde habían sido sometidos a un proceso de modernización. Entre noviembre y diciembre se realizaron en dos oportunidades maniobras, ejercicios de tiro y juegos de guerra en las afueras de Chilca, al sur de Lima, es muy probable que hayan participado los destructores de escolta.

Durante 1967 la escuadra realizó diversos ejercicios en la mar, pero con menos frecuencia, es probable que hayan participado nuestros destructores de escolta.

En febrero de 1968 la escuadra zarpó para realizar el operativo Alfa entre el Callao y Talara; es probable que hayan participado los destructores de escolta. Entre el 16 y el 23 de abril se desarrollo el operático Bravo entre 
San Juan y Pisco, en donde también es probable que hayan participado los destructores de escolta junto con el crucero Coronel Bolognesi, unidades aéreas de la aviación naval y de la Fuerza Aérea del Perú.

Entre enero y abril de 1970 la escuadra realizó los operativos ALFA y BRAVO, el primero para maniobras cerca de Salaverry y el segundo para tiro en Salinas, en donde es probable que hayan participado los destructores de escolta.

En 1971 todos los buques de la escuadra se hicieron a la mar para operativos en febrero, abril, mayo y junio. El 18 de agosto salieron el Aguirre y el Rodríguez junto con el crucero Almirante Grau, los destructores Villar y Guise, la cañonera torpedera Santillana además del petrolero Lobitos $_{93}$ para dar inicio a las operaciones UNITAS XI junto con los buques estadounidenses MacDonough $_{94}$, Bordelon ${ }_{95}$, Edward McDonell ${ }_{96}$ y Trumpetfish ${ }_{97}$; y los colombianos Almirante $\mathrm{Tono}_{98}$ y Córdoba ${ }_{99}$. La operación se desarrolló entre Talara y el Callao en una primera etapa, luego entre el 4 y 10 de septiembre se realizó entre el Callao e Ilo la segunda etapa.

Entre abril y noviembre de 1972 la escuadra realizó cuatro operativos encabezados por los cruceros Almirante Grau y Coronel Bolognesi, en el último se desarrollaron maniobras de tiro y navegación en Salinas, en donde es probable que hayan participado los destructores de escolta. En esta operación se contó con la presencia del Presidente del Comando Conjunto, comandantes generales y oficiales de las fuerzas armadas.

Entre febrero y marzo de 1973 se realizaron maniobras conjuntas en dos oportunidades en el área de San Juan encabezadas por los cruceros Almirante Grau y Coronel Bolognesi en donde es probable que hayan participado nuestros destructores de escolta.

El 7 de julio a la altura de Paita el Rodríguez junto con el crucero Coronel Bolognesi, el destructor Guise y el petrolero Lobitos, todos buques que estaban participando en el operativo JULIO, se encontraron con el nuevo crucero Almirante $\mathrm{Grau}_{100}$, recibiéndolo. Navegaron a Salinas el 9, aquí se encontraron con el buque escuela Independencia ${ }_{101}$, el Paita ${ }_{102}$, los submarinos Dos de Mayo y Abtao y las cañoneras torpederas Larrea y Sánchez Carrión; y todos salieron al Callao llegando el 11. 
El 4 de octubre se realiza la Revista Naval en conmemoración del $94^{\circ}$ aniversario del Combate naval de Angamos. En la revista participaron doce unidades de la escuadra; una de ellas fue el Rodríguez. La Revista la encabezó el crucero Almirante Grau, después del desfile se realizaron ejercicios y maniobras que iniciaron las cañoneras torpederas, los submarinos, y luego el Rodríguez realizó una demostración de lanzamiento de cargas de profundidad con el lanzador K-gun.

En 1974, el Aguirre fue dado de baja, siendo utilizado como blanco y hundido, en una prueba de tiro real con un misil Aérospatiale MM-38 Exocet, lanzado por el destructor Palacios ${ }_{103}$.

En agosto de 1975 la escuadra realizó maniobras y ejercicios de tiro, es muy probable que hayan participado los destructores de escolta Castilla y Rodríguez. El 6 de octubre se realiza la Revista Naval en conmemoración del $96^{\circ}$ aniversario del Combate naval de Angamos. En la revista que se llevó a cabo en las afueras del Callao participa el Rodríguez junto a los cruceros Almirante Grau y Capitán Quiñones ${ }_{73}$, los destructores Villar, Guise y Ferré ${ }_{104}$, los submarinos Dos de Mayo, Abtao, Arica ${ }_{105}$ e Islay $_{106}$ y el buque de desembarco Chimbote $_{107}$. A esta revista asistió el Presidente de la República general Francisco Morales Bermúdez, miembros del alto mando naval y autoridades civiles y militares.

En enero de 1976 el Rodríguez participa en el crucero de instrucción de cadetes junto a los cruceros Almirante Grau y Capitán Quiñones, los destructores Villar y Guise, los submarinos Abtao, Angamos, Arica, Islay y La Pedrera ${ }_{108}$. Partieron del Callao el 2 visitando Pisco, San Juan, Chala y Matarani.

En 1977 el Castilla fue transferido a Iquitos, convertido a pontón, sirvió como centro de entrenamiento flotante. En este año se realizaron también los operativos Tauro, Spica, Alpheca y Fanning en las que participó la mayor parte de los buques de la escuadra, en donde es probable que haya participado el Rodríguez con los cruceros Almirante Grau, Coronel Bolognesi y Capitan Quiñones y otras unidades de la escuadra. 
Entre el 14 de enero y el 25 de febrero de 1979 el Rodríguez junto con el crucero Almirante Grau, la fragata Carvajal ${ }_{109}$, los destructores Villar y García y García ${ }_{110}$, además de los submarinos Dos de Mayo y Angamos participó de la primera etapa del crucero de instrucción por el litoral con cadetes de primer y tercer año de la Escuela Naval del Perú, los puertos visitados fueron puerto General San Martín, San Juan, Ilo y Matarani.

El 5 de octubre se realiza la Revista Naval en conmemoración del centenario del Combate naval de Angamos. En la revista participaron la mayor parte de los buques de la escuadra, que sumaron 35 buques, en donde es probable que haya participado el Rodríguez. A este importante acto asistió el Presidente de la República general Francisco Morales Bermúdez, los miembros de la Junta Militar de Gobierno y al alto mando naval. Este año se le dio de baja al BAP Castilla (DE-61).

Desde 1980 el Rodríguez se convierte en buque nodriza de submarinos con el numeral de casco AMS-163, sirviendo como unidad generadora de energía y alojamiento para las tripulaciones de los submarinos.

El 29 de enero de 1981 el Rodríguez zarpa en compañía del crucero Almirante Grau, los destructores Villar y Palacios, así como el petrolero Zorritos $_{111}$ a Paita, donde llegan el 31, al día siguiente, partieron a Bayovar, retornando al Callao el día 4 de febrero. Esta operación se realizó como parte de las acciones navales producidas durante el conflicto con Ecuador de 1981 conocido también como Falso Paquisha.

Hacia el año 1982 el casco de ex BAP Castilla aún estaba en uso para instrucción en Iquitos.

Para el año 1987 al Rodríguez se le habían retirado algunas estructuras y el armamento, siguiendo desempeñándose como buque nodriza de submarinos pasando a la condición de reserva.

Entre los años 1990 y 1991 el Rodríguez sigue figurando en el listado de unidades de la Marina de Guerra del Perú como buque nodriza de submarinos. 


\section{LOS DESTRUCTORES CANNON PRESERVADOS COMO BUQUES MUSEO EN LA ACTUALIDAD}

En la actualidad dos destructores de la clase Cannon han sido preservados como buques museo, uno de ellos en América del Sur, nos referimos al brasileño Bauru ex USS McAnn (DE-179), después de haber sido dado de baja el 17 de septiembre de 1981 se ha preservado como Navio-Museu en Rio de Janeiro desde el 21 de julio de 1982.

Ubicación:

Espaço Cultural da Marinha

Boulevard Olímpico, Praça XV

Rio de Janeiro, Rio de Janeiro, Brasil.

Teléfono: (21) 2532-5992 - 2233-9165

www.marinha.mil.br/dphdm/navio-bauru

El otro buque de la clase Cannon excelentemente preservado como buque museo es el USS Slater (DE-766) que regresó a los Estados Unidos después de haber sido transferido a la armada griega en 1951 donde permaneció en servicio como Aetos hasta 1991. Después de su restauración, fue exhibido en el Intrepid Sea, Air \& Space Museum, en el río Hudson, Nueva York hasta 1997. Hoy se conserva en Albany, Nueva York sitio de su exposición permanente.

Ubicación:

Destroyer Escort Historical Museum

USS SLATER DE766

Broadway and Quay streets

Albany, New York 12202

Teléfono: (518) 4311943

www.ussslater.org 


\section{CITAS BIBLIOGRÁFICAS}

1 El USS Cannon (DE-99) fue el destructor de escolta que dio inicio a la clase homónima. Fue puesto en quilla el 14.11.1942, lanzado el 25.05.1943 y comisionado el 26.09.1943. Descomisionado y transferido a la Marinha do Brasil el 19.12.1944 en donde se le denominó Baependi (D17). Fue dado de baja en 1975.

2 El BRP Rajah Humabon (FF-11) es un destructor de escolta de la clase Cannon. Originalmente USS Atherton (DE-169) de la armada estadounidense. Fue puesto en quilla el 14.01.1943, lanzado el 27.05.1943 y comisionado el 29.08.1943. Descomisionado el 10.12.1945. Transferido a la armada japonesa el 14.06.1955 en donde se le denominó Hatsuhi(DE-263) hasta su baja en 1975 siendo devuelto a los Estados Unidos. Transferido a la armada filipina el 23.12.1978 fue enviado a la República de Corea para una modernización. Comisionado el 27.02.1980. Fue descomisionado en 1993. Vuelto a comisionar en enero de 1996, fue reclasificado como fragata de patrulla. Actualmente se encuentra en servicio.

3 El USS Riddle (DE-185) fue un destructor de escolta de la clase Cannon. Fue puesto en quilla el 29.07.1943, lanzado el 17.10.1943 y comisionado el 17.11.1943. Descomisionado el 08.06.1946. Fue transferido a la Marine Nationale el 12.08.1950 en donde se le denominó Kabyle (F718) hasta su baja en 1959 siendo posteriormente desguazado en 1965.

4 Joseph Bangust (Niles, Ohio, Estados Unidos, 30.05.1915-Isla Lugus, Sulu, Filipinas, 27.12.1941). Joseph Bangust se alistó en la armada de los Estados Unidos el 16 de noviembre de 1938 en la Estación Naval de Entrenamiento de San Diego, California. Fue promovido a marinero de $2^{\circ}$ clase el 16 de diciembre de 1938 siendo transferido a la Segunda Ala de Patrulla en junio de 1939, y, dentro de esta unidad, a la Escuadrilla de Patrulla 21 (VP-21) en julio. Fue transferido junto con su escuadrón que se desplegó a Filipinas con sus hidrocanoas Consolidated PBY-4 Catalina, y en diciembre de 1939 recibió ascenso a marinero de $1^{\circ}$ clase. Luego, fue ascendido dos veces; a mecánico de aviación de $3^{\circ}$ clase, en mayo de 1940 y a mecánico de aviación de $2^{\circ}$ clase en febrero de 1941. El comienzo de las hostilidades entre los Estados Unidos y Japón en diciembre de 1941 encontró a Joseph Bangust asignado al VP-101, ya que VP-21 había sido reorganizado. El 26 de diciembre de 1941 seis hidrocanoas PBY-4 Catalina partieron de su base de avanzada en Ambon (Indias Orientales holandesas) a las 23:00 horas para atacar a la fuerza de desembarco 
japonesa en el puerto de Jolo en el archipiélago de Sulu. Bangust voló como segundo mecánico y artillero en la nave comandada por el alférez Elwyn L. Christman del USNR. Al llegar a su objetivo la artillería antiaérea japonesa emplazada en la costa atacó a los PBY-4 cuando sobrevolaban el puerto, pero terminaron su ataque cuando un grupo de cazas Mitsubishi A6M2 Zero aparecieron para interceptar a los atacantes. Bangust y su compañero, el también mecánico de aviación Andrew Kenneth Waterman, el otro artillero, derribaron al primer Zero japonés de la guerra. El ataque se llevó a cabo; Christman lanzó sus bombas, dirigiéndose luego al oeste, a lo largo del archipiélago de Sulu. Un Zero lo siguió atacándolo persistentemente, pero Christman frustró al atacante dirigiéndose hacia él, forzándolo a romper sus ataques. Un duelo mortal se produjo, por desgracia, un proyectil del Zero perforó el depósito de combustible, lanzando gasolina al compartimento de los mecánicos. En otro ataque, el Zero logró impactar nuevamente la aeronave encendiendo el combustible. Bangust cegado, y habiendo sufrido quemaduras de tercer grado en cara, manos y cuello, fue rescatado por el radioperador de $2^{\circ}$ clase, P. H. Landers. Mientras tanto, el alférez Christman, con su avión en llamas tuvo que amerizar, abandonando la aeronave. Landers, con menos lesiones que Bangust, ayudó en los esfuerzos por nadar de este último hacia la isla Lugus. Alrededor del mediodía del 27 de diciembre, Landers vio por detrás a su compañero herido, pero vio sólo un chaleco salvavidas vacío; aparentemente Bangust se había deslizado y se había ahogado. Por su "valentía y exitoso ataque" y por su "heroísmo extraordinario" durante la acción sobre Jolo, Joseph Bangust fue galardonado póstumamente con la Cruz de la Marina.

5 El USS Reynolds (DE-42) fue un destructor de escolta de la clase Evarts. Fue puesto en quilla el 12.01.1943, lanzado el 01.08.1943 y comisionado el 08.11.1943. Descomisionado el 05.12.1945 fue vendido para su desguace el 28.04.1947.

6 El USS Pastores (AF-16) fue un buque almacén. Fue lanzado en 1913, comisionado el 06.05.1918. Descomisionado el 08.10.1919, al inicio de la II Guerra Mundial fue reincorporado el 13.02.1942, finalmente fue descomisionado el 14.03.1946 fue vendido para su desguace el 19.12.1946.

7 El USS Ajax (AR-6) fue un buque de reparaciones de la clase Vulcan. Fue puesto en quilla el 07.05.1941, lanzado el 22.08.1942 y comisionado el 30.10.1943. Descomisionado el 31.12.1986 fue vendido para su desguace el 23.05.1997. 
8 El USS Wadleigh (DD-689) fue un destructor de la clase Fletcher. Fue puesto en quilla el 05.04.1943, lanzado el 07.08.1943 y comisionado el 19.10.1943. Descomisionado el 28.06.1962. Fue transferido a la Armada de Chile el 26.07.1962 en donde se le denominó Blanco Encalada (14) hasta su baja en 1982. Fue hundido el 28.09.1991 por el USS O'Bannon (DD987) un destructor de la clase Spruance con un misil RGM-84 Harpoon en el marco de la operación UNITAS XXXII.

9 El USS Greiner (DE-37) fue un destructor de escolta de la clase Evarts. Fue puesto en quilla el 07.09.1942, lanzado el 20.05.1943 y comisionado el 18.08.1943. Descomisionado el 19.11.1945 fue vendido para su desguace el 10.02.1946.

10 El USS Caliente (AO-53) fue un petrolero de la clase Cimarron. Fue puesto en quilla el 02.01.1943, lanzado el 25.08.1943 y comisionado el 12.10.1943. Descomisionado el 13.11.1973 fue vendido para su desguace el 05.04.1974.

11 El USS Fleming (DE-32) fue un destructor de escolta de la clase Evarts. Fue puesto en quilla el 24.12.1942, lanzado el 16.06.1943 y comisionado el 18.09.1943. Descomisionado el 15.11.1945 fue vendido para su desguace el 29.01.1948.

12 El USS Anacapa (AG-49) fue un buque transporte. Originalmente COOS BAY. Fue lanzado en 15.02.1919. Adquirido al inicio de la II Guerra Mundial el 20.06.1942, fue comisionado el 31.08.1942. Fue descomisionado el 21.03.1946, fue transferido a la Comisión Marítima y sirvió como mercante primero con su nombre original y luego como GEORGE OLSON hasta que naufragara en el río Columbia el 30.01.1964 cuando transportaba madera.

13 El USS Eisele (DE-75) fue una fragata de la clase Captain. Fue puesto en quilla el 03.05.1943, lanzado el 26.07.1943 y comisionado el 17.10.1943. Fue transferido a la Royal Navy en donde se le denominó HMS BICKERTON (K466). Fue hundido al ser torpedeado por submarino alemán U-354 a la 01:22 horas del 22.08.1944 al noroeste del cabo norte en el Mar de Barents.

14 El USS LST-29 fue un buque de desembarco de la clase LST-1. Fue puesto en quilla el 08.01.1943, lanzado el 17.05.1943 y comisionado el 10.07.1943. Descomisionado el 11.03.1946 fue vendido para su desguace el 17.06.1946. 
15 El USS Kenmore (AK-221) fue un buque transporte de la clase Crater, originalmente fue el SS James H. McClintock Fue puesto en quilla el 08.05.1943, lanzado el 30.05.1943 y comisionado el 14.11.1943. Descomisionado el 01.02.1946 fue vendido para su desguace el 13.02.1973.

16 El USS De Grasse (AK-223) fue un buque transporte de la clase Crater, originalmente fue el SS Nathaniel J. Wyeth. Fue puesto en quilla el 31.01.1943, lanzado el 24.02.1943. Adquirido el 28.10.1943 y comisionado el 08.11.1943. Descomisionado el 28.03.1946 fue vendido para su desguace el 10.02.1970.

17 El USS Kaskaskia (AO-27) fue un petrolero de la clase Cimarron. Fue lanzado el 29.09.1939. Adquirido el 22.10.1940 y comisionado el 29.10.1940. Descomisionado En diciembre de 1969 fue vendido para su desguace en septiembre de 1970 .

18 El USS LST-119 fue un buque de desembarco de la clase LST-1. Fue puesto en quilla el 12.05.1943, lanzado el 28.07.1943 y comisionado el 01.09.1943. Descomisionado el 13.05.1948 fue vendido para su desguace el 17.05.1948.

19 El USS Capable (AM-155) fue un dragaminas de la clase Admirable. Fue puesto en quilla el 12.05.1942, lanzado el 16.10.1942 y comisionado el 05.12.1943. Descomisionado el 17.08.1945. Fue transferido el mismo día a la armada soviética en donde se le denominó T-339. Fue dado de baja en 1960. 20 El USS ATR-44 fue un remolcador de flota de la clase Sotoyomo. Fue puesto en quilla el 19.10.1942, lanzado el 17.11.1942 y comisionado el 10.06.1943. Descomisionado el 05.08.1946. Fue transferido a la Armada de Chile el 19.09.1947 en donde se le denominó Lautaro (PP-62). Fue dado de baja en 1991. Fue transferido a la Armada Nacional de Uruguay el 11.06.1991 en donde se le denominó ROU 22 San José. Fue dado de baja el 25.01.1993.

21 El USS Grouper (SS-214) fue un submarino de la clase Gato. Fue puesto en quilla el 28.12.1940, lanzado el 27.10.1941 y comisionado el 12.02.1942. Descomisionado el 02.12.1968 fue vendido para su desguace el 11.08.1970.

22 El USS Lackawanna (AO-40) fue un petrolero de la clase Kennebec. Fue puesto en quilla el 27.12.1941, lanzado el 16.05.1942. Adquirido el 20.06.1942 y comisionado el 10.07.1942. Descomisionado el 14.02.1946, fue transferido a la Comisión Marítima el 01.07.1946, 
finalmente fue vendido en 1947 a la compañía petrolera Mobil Oil en donde navegó como Tatarrax hasta 1962, luego como Thomas A. hasta 1965 y luego como Padre Island hasta que finalmente fue desguazado en 1967.

23 El RO-42 fue un submarino de la clase Kaychu. Fue puesto en quilla el 27.04.1942, lanzado el 25.10.1943 y comisionado el 31.08.1943. Hundido por el USS Bangust (DE-739) el 10.06.1944.

24 El USS YMS-282 fue un dragaminas de la clase BYMS. Construido para la Royal Navy bajo la ley de préstamo y arriendo en donde se le denominó HMS BYMS-2282. Fue puesto en quilla el 15.08.1942, lanzado el 30.11.1942 y comisionado el 26.08.1943. Descomisionado el 28.08.1947. Fue transferido a la armada polaca el 18.04.1948 en donde se le denominó ORP MORS. Fue dado de baja el 08.01.1957.

25 El USS YMS-283 fue un dragaminas de la clase BYMS. Construido para la Royal Navy bajo la ley de préstamo y arriendo en donde se le denominó HMS BYMS-2203. Fue puesto en quilla el 15.01.1942, lanzado el 15.01.1943 y comisionado el 31.08.1943. Descomisionado el 13.12.1946. No se sabe de su final.

26 El USS SC-1364 fue un cazasubmarino de la clase Wooden. Fue construido en el astillero Peyton Co. No se sabe de su final.

27 El USS Capps (DD-550) fue un destructor de la clase Fletcher. Fue puesto en quilla el 12.06.1941, lanzado el 31.05.1942 y comisionado el 23.06.1943. Descomisionado el 15.01.1947. Fue transferido a la Real Armada Española el 15.05.1957 en donde se le denominó Lepanto (D21) hasta su baja el 31.12.1985. Fue posteriormente desguazado.

28 El USS Neosho (AO-48) fue un petrolero de la clase Mattaponi. Fue puesto en quilla el 08.07.1941, lanzado el 23.12.1941. Adquirido el 04.08.1942 y comisionado el 16.09.1942. Descomisionado el 13.12.1945, fue transferido a la Comisión Marítima el 30.06.1946, finalmente fue vendido en 1947 a la compañía petrolera Mobil Oil en donde navegó como Tascalusa hasta 1963, luego como Ascalusa hasta que finalmente fue desguazado en febrero de 1964.

29 El USS Whitman (DE-24) fue un destructor de escolta de la clase Evarts. Fue puesto en quilla el 07.09.1942, lanzado el 19.06.1943 y comisionado el 03.07.1943. Descomisionado el 16.11.1945, fue vendido para su desguace el 31.01.1947. 
30 El USS John D. Henley (DD-553) fue un destructor de la clase Fletcher. Fue puesto en quilla el 21.07.1941, lanzado el 15.11.1942 y comisionado el 02.02.1944. Descomisionado el 30.04.1946, fue vendido para su desguace el 31.01.1970.

31 El USS Fair (DE-35) fue un destructor de escolta de la clase Evarts. Fue puesto en quilla el 24.02.1943, lanzado el 27.07.1943 y comisionado el 23.10.1943. Descomisionado el 17.11.1945, Fue transferido al ejército estadounidense el 20.05.1947 para prestarlo a Canadá. Devuelto en 1948, fue desguazado en 1949.

32 El USS Acree (DE-167) fue un destructor de escolta de la clase Cannon. Fue puesto en quilla el 30.11.1942, lanzado el 09.05.1943 y comisionado el 19.07. 1943. Descomisionado el 01.04.1946. Dado de baja el 01.07.1972, fue vendido para su desguace el 19.07.1973.

33 El USS Mascoma (AO-83) fue un petrolero de la clase Escambia. Fue puesto en quilla el 31.01.1943, lanzado el 31.05.1943 y comisionado el 03.02.1944. Descomisionado el 17.12.1945. Fue dado de baja el 08.01.1946. Fue transferido a la Comisión Marítima el 27.06.1946, fue nuevamente comisionado el 04.02.1948, dado de baja el 18.06.1959 fue vendido a la compañía Seatrain Lines Inc, luego fue vendido a la compañía Hudson Waterways Corporation el 04.11.1966 en donde navegó como Seatrain Oregon, luego fue vendido a la compañía Transwestern Associates Inc en donde navegó como Transchamplain en septiembre de 1967. En 1974 fue transferido a Matson Inc, hasta que finalmente fue vendido para su desguace en 1980.

34 El USS Niobrara (AO-72) fue un petrolero de la clase Chiwawa. Fue puesto en quilla el 29.06.1942, lanzado el 28.11.1942. Adquirido y comisionado el 13.03.1943. Descomisionado el 24.09.1946. Fue nuevamente comisionado el 05.02.1951, descomisionado el 30.11.1954 fue otra vez comisionado el 14.12.1956 y finalmente descomisionado el 12.11.1957, fue dado de baja el 01.02.1959. Finalmente fue vendido para su desguace el 22.03.1982.

35 El USS Swearer (DE-186) fue un destructor de escolta de la clase Cannon. Fue puesto en quilla el 12.08.1943, lanzado el 31.10.1943 y comisionado el 23.11.1943. Descomisionado el 25.02.1946. Dado de baja el 20.10.1950, Fue transferido a la Marine Nationale el 16.09.1950 en donde se le denominó Bambara (F-719) hasta su baja en 1959.

36 El USS Nehenta Bay (CVE-74) fue un portaviones de escolta de la clase Casablanca. Fue lanzado el 28.11.1943 y comisionado el 03.01.1944. 
Descomisionado el 15.05.1946. Finalmente fue vendido para su desguace el 29.06.1960.

37 El USS Aylwin (DD-355) fue un destructor de la clase Farragut. Fue puesto en quilla el 23.09.1933, lanzado el 10.07.1934 y comisionado el 01.03.1935. Descomisionado el 16.10.1945. Dado de baja el 01.11.1945, fue vendido para su desguace el 20.12.1946.

38 El USS Spence (DD-512) fue un destructor de la clase Fletcher. Fue puesto en quilla el 18.05.1942, lanzado el 27.10.1942 y comisionado el 08.01.1943. Se hundió a causa del tifón Cobra el 18.12.1944.

39 El USS Hull (DD-350) fue un destructor de la clase Farragut. Fue puesto en quilla el 07.03.1933, lanzado el 31.07.1934 y comisionado el 11.01.1935. Se hundió a causa del tifón Cobra el 18.12.1944.

40 El USS Kyne (DE-744) fue un destructor de escolta de la clase Cannon. Fue puesto en quilla el 16.04.1943, lanzado el 15.08.1943 y comisionado el 04.04.1944. Descomisionado el 14.06.1946. Fue nuevamente comisionado el 21.11.1950, descomisionado el 17.06.1960. Dado de baja el 01.08.1972, fue vendido para su desguace el 01.11.1973.

41 El USS Chikaskia (AO-54) fue un petrolero de la clase Cimarron. Fue lanzado el 02.10.1942. Adquirido el 10.01.1943 y comisionado el 10.11.1943. Descomisionado el 18.12.1969. Fue dado de baja el 01.12.1976 y vendido para su desguace en septiembre el 26.05.1982.

42 El USS Neches (AO-47) fue un petrolero de la clase Mattaponi. Fue puesto en quilla el 12.06.1941, lanzado el 11.10.1941. Adquirido el 20.07.1941 y comisionado el 16.09.1942. Descomisionado el 10.07.1950, Fue nuevamente comisionado el 03.01.1951, descomisionado en junio de 1955. Fue otra vez comisionado el 24.11.1961 y descomisionado en 1970. Dado de baja el 01.10.1970, fue vendido para su desguace el 01.12.1973.

43 El USS Tomahawk (AO-88) fue un petrolero de la clase Escambia. Fue puesto en quilla el 01.06.1943, lanzado el 10.10.1943 y comisionado el 16.04.1944. Descomisionado el 06.01.1946. Fue dado de baja el 21.01.1946. Fue arrendado a la American Petroleum Transportation el 17.10.1947, regresó a la armada estadounidense el 28.02.1948. Fue transferido a la Comisión Marítima el 01.09.1961. Fue vendido a la compañía Hudson Waterways Corporation el 21.07.1966 convertido a buque portacontenedores en mayo de 1967, renombrado SS Maine el 08.08.1978, vuelto a comisionar para la operación Tormenta del 
Desierto el 28.08.1990, pasó a la reserva el 12.06.1991. Fue vendido para su desguace el 13.03.2008.

44 El USS Merrimack (AO-37) fue un petrolero de la clase Kennebec. Fue puesto en quilla el 12.09.1940 como SS Caddo, lanzado el 01.07.1941. Adquirido el 31.12.1941. Renombrado Merrimack el 09.01.1942. Fue comisionado el 04.02.1942. Descomisionado el 08.02.1950. Fue nuevamente comisionado el 06.12.1950, descomisionado el 20.12. 1954. Dado de baja el 04.02.1959, fue vendido para su desguace el 29.03.1982.

45 El USS Crowley (DE-303) fue un destructor de escolta de la clase Evarts. Fue puesto en quilla el 24.05.1943, lanzado el 22.09.1943 y comisionado el 25.03.1944. Descomisionado el 03.12.1945, fue de baja el 19.12.1945 y vendido para su desguace el 21.12.1946.

46 El USS Admiralty Islands (CVE-99) fue un portaviones de escolta de la clase Casablanca. Fue puesto en quilla el 26.02.1944, lanzado el 10.05.1944 y comisionado el 13.06.1944. Descomisionado el 24.04.1946. Fue dado de baja el 08.05.1946, finalmente fue vendido para su desguace el 02.01.1947.

47 El USS Hollandia (CVE-97) fue un portaviones de escolta de la clase Casablanca. Fue puesto en quilla el 12.02.1944, lanzado el 28.04.1944 y comisionado el 01.06.1944. Descomisionado el 17.01.1947.Fue dado de baja el 01.04.1960, finalmente fue vendido para su desguace el 31.12.1960.

48 El USS Thetis Bay (CVE-90) fue un portaviones de escolta de la clase Casablanca. Fue puesto en quilla el 22.12.1943, lanzado el 16.03.1944 y comisionado el 12.04.1944. Descomisionado y dado de baja el 01.03.1964, finalmente fue vendido para su desguace en diciembre de 1964.

49 El USS Roi (CVE-103) fue un portaviones de escolta de la clase Casablanca. Fue puesto en quilla el 22.03.1944, lanzado el 02.06.1944 y comisionado el 06.07.1944. Descomisionado el 09.05.1946. Fue dado de baja el 21.05.1946, finalmente fue vendido para su desguace el 31.12.1946.

50 El USS Mc Clelland (DE-750) fue un destructor de escolta de la clase Cannon. Fue puesto en quilla el 21.07.1943, lanzado el 28.11.1943 y comisionado el 19.09.1944. Descomisionado el 15.05.1946. Fue nuevamente comisionado el 14.07.1950, descomisionado el 12.09.1960. Dado de baja el 01.08.1972, fue vendido para su desguace el 01.11.1973. 
51 El USS Thorn (DE-647) fue un destructor de escolta de la clase Gleaves. Fue puesto en quilla el 15.11.1942, lanzado el 28.02.1943 y comisionado el 01.04.1943. Descomisionado el 06.05.1946. Dado de baja el 01.07.1971, fue utilizado como blanco y hundido el 22.08 .1974 por aviones del portaaviones USS Saratoga (CV-60).

52 El I-14 fue un submarino de la clase AM. Fue puesto en quilla el 18.05.1943, lanzado el 14.03.1944 y comisionado el 14.03.1945. Rendido al final de la II Guerra Mundial el 28.08.1945. Fue hundido como blanco en Oahu, Hawai el 28.05.1946.

53 El USS Murray (DD-576) fue un destructor de la clase Fletcher. Fue puesto en quilla el 16.03.1942, lanzado el 16.08.1942 y comisionado el 20.04.1943. Descomisionado el 27.03.1946. Fue nuevamente comisionado el 02.01.1951, descomisionado y dado de baja el 01.06.1965 Descomisionado el 15.05.1946. Fue nuevamente comisionado el 14.07.1950, descomisionado el 12.09.1960. Dado de baja el 01.08.1972, fue vendido para su desguace el 16.08.1966.

54 El USS Proteus (AS-19) fue un buque nodriza de submarinos de la clase Fulton. Fue puesto en quilla el 15.09.1941, lanzado el 12.11.1942 y comisionado el 31.01.1944. Descomisionado el 26.09.1947. Fue nuevamente comisionado el 08.07.1960, descomisionado y dado de baja el 30.09.1992. Fue nuevamente comisionado en 1994, descomisionado el 30.09.1999. Dado de baja el 13.03.2001, fue vendido para su desguace el 01.07.2007.

55 El USS Challenge (ATA-201) fue un remolcador de flota de la clase Sotoyomo. Fue puesto en quilla el 03.08.1944, lanzado el 23.09.1944 y comisionado el 22.11.1944. Dado de baja el 01.09.1962. Fue vendido para su desguace a la compañía Defence Reutilization and Marketing Service el 01.10.1976 que lo revendió a en 1978 a la compañía Marine Power \& Equipment que lo nombró Marine Commander; vendido luego a la compañía Alaska Oregon Offshore Marine que mantuvo su nombre; Vendido en 2007 a la compañía West African Co, que lo nombró Saje Commander.

56 El USS Tunica (ATA-178) fue un remolcador de flota de la clase Sotoyomo. Fue puesto en quilla el 10.05.1944, lanzado el 15.06.1944 y comisionado el 15.09.1944. Descomisionado el 23.12.1947. Dado de baja el 01.09.1962 fue transferido a la reserva de la armada hasta el 27.08.1986 en que fue enviado a Pearl Harbor como casco de entrenamiento para la 
unidad de salvataje, fue utilizado como blanco y hundido el 29.01.1999 durante los ejercicios

55 Andrew Kenneth Waterman (Lewis County, Kentucky, Estados Unidos, 20.12.1913-Isla Lugus, Sulu, Filipinas, 27.12.1941). Andrew Kenneth Waterman se alistó en la armada de los Estados Unidos el 21 de noviembre de 1932 en Buffalo, Nueva York. Después de su instrucción en la Estación de Entrenamiento Naval de Newport, Rhode Island, sirvió en los buques de la armada USS Nitro, USS Nuevo México y USS Relief, luego se entrenó en aviación en la base aérea de Coco Solo. Fue ascendido a mecánico de aviación de $3^{\circ}$ clase en 1935 extendiendo su alistamiento en noviembre de 1936 sirviendo en el escuadrón utilitario $1^{\circ}$ hasta mediados de 1939 en que fue transferido a la Escuadrilla de Patrulla 21 (VP-21), realizando de inmediato un vuelo transpacífico de Hawai a Filipinas en las hidrocanoas Consolidated PBY-4 Catalina con el objetivo de reforzar las patrullas de la flota asiática desde Cavite y Olonpago. Por su papel en esta misión, Waterman recibió una felicitación del comandante del ala de patrulla, contralmirante Arthur L. Bristol Jr. el 02.10.1939 que se expresaba con estas palabras: "a través de la planificación, atención al detalle y excepcional habilidad que indicaba un alto grado de eficiencia, fundamental en el éxito de esta importante misión". Transferido al VP-1en septiembre de 1940 y luego a la Patrulla 10 con la que realizó misiones de reconocimiento y patrulla desde la base de hidroaviones de Cavite en Sangley Point o desde Olonpago hasta el estallido de la guerra el 08.12.1941, a partir de este momento la Patrulla 10 tuvo que realizar sus misiones con gran dificultad por los constantes ataques japoneses. El 26 de diciembre de 1941 seis hidrocanoas PBY-4 Catalina partieron de su base de avanzada en Ambon (Indias Orientales holandesas) a las 23:00 horas para atacar a la fuerza de desembarco japonesa en el puerto de Jolo en el archipiélago de Sulu. Waterman, que había sido ascendido mecánico de aviación de $1^{\circ}$ clase voló como primer mecánico y artillero en la nave comandada por el alférez Elwyn L. Christman del USNR. Al llegar a su objetivo la artillería antiaérea japonesa emplazada en la costa atacó a los PBY-4 cuando sobrevolaban el puerto, pero terminaron su ataque, cuando un grupo de cazas Mitsubishi A6M2 Zero aparecieron para interceptar a los atacantes. Waterman y su compañero, el también mecánico de aviación Joseph Bangust, el otro artillero, derribaron al primer Zero japonés de la guerra. El ataque se llevó a cabo; Christman lanzó sus bombas, dirigiéndose luego al oeste, a lo largo del archipiélago de Sulu. Un Zero lo siguió atacándolo persistentemente, pero Christman frustró al atacante dirigiéndose hacia él, 
forzándolo a romper sus ataques. Un duelo mortal se produjo, por desgracia, un proyectil del Zero perforó el depósito de combustible, lanzando gasolina al compartimento de los mecánicos. En otro ataque, el Zero logró impactar nuevamente la aeronave encendiendo el combustible. Mientras tanto, el alférez Christman, con su avión en llamas tuvo que amerizar. Waterman había sido herido mortalmente, posiblemente en el último ataque del Zero, todos los sobrevivientes abandonaron la aeronave para nadar a la isla; lamentablemente no hubo tiempo de sacar el cuerpo de Waterman. Por su "heroísmo y coraje extraordinario" durante la acción sobre Jolo, Andrew Kenneth Waterman fue galardonado póstumamente con la Cruz de la Marina.

56 El USS Nassau (CVE-99) fue un portaviones de escolta. Fue puesto en quilla el 27.11.1941, lanzado el 04.04.1942 y comisionado el 20.08.1942. Descomisionado el 28.10.1946. Fue dado de baja el 01.03.1959, finalmente fue vendido para su desguace en junio de 1961 .

57 El USS Bowers (DE-637) fue un destructor de escolta de la clase Buckley. Fue puesto en quilla el 28.03.1943, lanzado el 31.10.1943 y comisionado el 27.01.1944. Descomisionado el 10.02.1947. Nuevamente comisionado el 06.02.1951 y descomisionado el 18.12.1958. Fue transferido a la Armada filipina el 21.04.1961 en donde se le denominó BRP Rajah Soliman (D-66) hundido por un tifón en el astillero nacional de Bataan fue reflotado en 1964 y vendido para su desguace el 31.01.1966.

58 El USS Patuxent (AO-44) fue un petrolero de la clase Mattaponi. Fue puesto en quilla el 05.03.1942, lanzado el 25.07.1942. Adquirido el 28.09.1942. Fue comisionado el 22.10.1942. Descomisionado el 21.02.1946. Dado de baja el 12.03.1946. Fue vendido a la compañía Sabine Transit Co el 18.07.1947 renombrado SS David D. Irwing, vendido luego a la compañía Delmor Corporation que mantuvo su nombre el 21.09.1961; Vendido a la compañía Union Oil Co el 09.03.1981. Vendido a la compañía American Fairwind Inc. el 23.07.1985 que lo denominó SS Fairwind. Fue vendido para su desguace el 05.12.1985.

59 El USS Dewey (DD-349) fue un destructor de la clase Farragut. Fue puesto en quilla el 16.12.1932, lanzado el 28.07.1934 y comisionado el 04.10.1934. Descomisionado el 19.10.1945. Dado de baja el 01.11.1945, fue vendido para su desguace el 20.12.1946.

60 El USS Attu (CVE-102) fue un portaviones de escolta de la clase Casablanca. Fue puesto en quilla el 16.03.1944, lanzado el 27.05.1944 y 
comisionado el 30.06.1944. Descomisionado el 08.06.1946. Fue dado de baja el 03.07.1946, finalmente fue vendido para su desguace el 03.01.1947.

61 El USS MISSOURI (BB-63) fue un acorazado de la clase Iowa. Fue puesto en quilla el 06.01.1941, lanzado el 29.01.1944 y comisionado el 11.06.1944. Descomisionado el 26.02.1955. Nuevamente comisionado el 10.05.1986 y descomisionado el 31.03.1992. En su cubierta se firmó la rendición de Japón, hecho que puso punto final a la II Guerra Mundial. Desde 1998 se encuentra en Pearl Harbor como buque museo.

62 Luther Dayton Weaver (Morrilton, Arkansas, Estados Unidos 20.07.1920-Pearl Harbor, Hawai, Estados Unidos 07.12.1941). Luther Dayton Weaver se alistó en la armada de los Estados Unidos el 12 de julio de 1940 en San Diego, California. Después de su instrucción básica en esta misma unidad, fue transferido a la Estación Aeronaval de Kaneohe Bay, Hawai, con el Escuadrón de Patrulla 11 de la $2^{\circ}$ Ala de Patrulla el 19 de septiembre. Fue ascendido a marinero de $1^{\circ}$ clase el 01.08.1941. Al amanecer del 7 de diciembre de 1941 se produjo el ataque sorpresa a Pearl Harbor por las fuerzas japonesas, Weaver murió en acción defendiendo su base ante el ataque enemigo. Por su "acción rápida y eficiente y total desprecio del peligro personal en el esfuerzo por repeler el ataque a la Estación Aérea Naval de bahía de Kaneohe", Luther Dayton Weaver recibió el elogio del Comandante en Jefe de la Flota del Pacífico. Waterman fue galardonado póstumamente con la Cruz de la Marina y el Corazón Púrpura.

63 El I-400 fue un submarino de la clase homónima. Fue puesto en quilla el 18.01.1943, lanzado el 18.01.1944 y comisionado el 30.12.1944. Capturado por el USS Blue (DD-744) el 19.08.1945, fue descomisionado el 15.09.1945. Fue hundido como blanco en Hawai por el submarino USS Trumpetfish (SS-425) el 04.06.1946.

64 El BAP R-1 fue un submarino de la clase R. Fue puesto en quilla el 25.02.1925, lanzado el 12.07.1926 y comisionado el 02.07.1926. Fue dado de baja el 29.10.1958.

65 El BAP R-2 fue un submarino de la clase R. Fue puesto en quilla el 25.02.1925, lanzado el 29.04.1926 y comisionado el 02.07.1926. Fue dado de baja el 29.10.1958.

66 El BAP R-3 fue un submarino de la clase R. Fue lanzado el 21.04.1928 y comisionado el 07.07.1928. Fue dado de baja el 29.10.1958.

67 El BAP R-4 fue un submarino de la clase R. Fue lanzado el 10.05.1928 y comisionado el 01.07.1928. Fue dado de baja el 29.10.1958. 
68 El BAP Teniente Palacios (FE-65) fue una fragata de la clase River. Originalmente HMCS St. Pierre (K680) de la armada canadiense. Fue puesta en quilla el 30.06.1943, lanzada el 01.12.1943 y comisionada el 28.08.1944. Descomisionada el 22.11.1945. Fue transferida a la Marina de Guerra del Perú en 1947 en donde se le denominó Teniente Palacios (FE2). En 1953 fue renombrada PALACIOS. Fue dada de baja en 1966.

69 El BAP Teniente Ferré (FE-66) fue una fragata de la clase River. Originalmente HMCS Poundmaker (K675) de la armada canadiense. Fue puesta en quilla el 29.01.1944, lanzada el 21.04.1944 y comisionada el 17.09.1944. Descomisionada el 25.11.1945. Fue transferida a la Marina de Guerra del Perú en 1947 en donde se le denominó Teniente Ferré (FE-3). En 1953 fue renombrada Ferré. Fue dada de baja en 1966.

70 El BAP Teniente Gálvez (FE-1) fue una fragata de la clase Tacoma. Originalmente USS Woonsocket (PF-32) de la armada estadounidense. Fue puesta en quilla el 12.08.1943, lanzada el 27.09.1943 y comisionada el 01.09.1944. Descomisionada el 16.03.1946. Fue transferida al US Coast Guard el 16.03.1946 en donde se le denominó USCGC Woonsocket y Descomisionada el 18.09.1946. Fue transferida a la Marina de Guerra del Perú en 1948 en donde se le denominó Teniente Gálvez (F-1), más adelante fue renombrada Gálvez (FE-1). Fue dada de baja en 1961.

71 El BAP Tiburón (5) fue un submarino de la clase S. puesto en quilla el 12.05.1952, lanzado el 27.10.1953 y comisionado el 01.03.1954. Renombrado Abtao el 29.03.1957. Fue dado de baja el 09.03.2000. Desde 2004 es buque museo en el Callao.

72 El BAP Lobo (6) fue un submarino de la clase S. puesto en quilla el 12.05.1952, lanzado el 06.02.1954 y comisionado el 14.06.1954. Renombrado Dos de Mayo en abril de 1957.Fue dado de baja en 1999.

73 El BAP Almirante Grau (CL-81) fue un crucero ligero de la clase Colony. Originalmente HMS Newfoundland (C-59) de la Royal Navy. Fue puesto en quilla el 09.11.1939, lanzado el 19.12.1941 y comisionado el 21.01.1943. Descomisionado el 30.12.1959. Fue transferido a la Marina de Guerra del Perú el 30.12.1959. Renombrado Capitán Quiñones (CL-83) el 15.05.1973. Fue dado de baja en 02.05.1980.

74 El BAP Coronel Bolognesi (CL-82) fue un crucero ligero de la clase Colony. Originalmente HMS Ceylon (C-30) de la Royal Navy. Fue puesto en quilla el 27.04.1939, lanzado el 30.07.1942 y comisionado el 
13.07.1943. Descomisionado el 30.12.1959. Fue transferido a la Marina de Guerra del Perú el 29.01.1960. Fue dado de baja en 20.09.1982.

75 El BAP Iquique (44) fue un submarino de la clase S. Fue puesto en quilla el 27.10.1955, lanzado el 05.02.1957 y comisionado el 01.10.1957. Anteriormente se había denominado Merlín. Fue dado de baja el 26.10.1993.

76 El BAP Angamos (43) fue un submarino de la clase S. Fue puesto en quilla el 27.10.1955, lanzado el 05.02.1957 y comisionado el 01.07.1957. Anteriormente se había denominado ATÚN. Fue dado de baja el 06.09.1990.

77 Ver el capítulo correspondiente a esta nave en el presente trabajo.

78 Ver el capítulo correspondiente a esta nave en el presente trabajo.

79 El BAP Gálvez (M68) fue un dragaminas de la clase Auk. Originalmente USS Ruddy (AM-380) de la armada estadounidense. Fue puesto en quilla el 24.02.1944, lanzado el 29.10.1944 y comisionado el 28.04.1945. Descomisionado y transferido a la Marina de Guerra del Perú el 01.11.1960 en donde se le clasificó como corbeta. Fue dado de baja el 17.05.1974. Transferido al Servicio de Guardacostas en 1975. Fue dado de baja en 1981.

80 El BAP Diez Canseco (M69) fue un dragaminas de la clase Auk. Originalmente USS Shoveler (AM-382) de la armada estadounidense. Fue puesto en quilla el 01.04.1944, lanzado el 10.12.1944 y comisionado el 22.05.1945. Descomisionado el 05.11.1946. Vuelto a comisionar el 24 de julio de 1951 y descomisionado el 28.09.1956 Transferido a la Marina de Guerra del Perú el 01.11.1960 en donde se le clasificó como corbeta. Fue dado de baja el 17.05.1974.

81 El BAP Talara (ATP-53) fue un petrolero. Fue puesto en quilla en 1953, lanzado el 20.10.1954 y comisionado el 31.12.1954. Dado de baja el 27.12.1968. Vendido a la compañía P.G. \& M. Castaldi de Livorno que lo renombró Punta Ala. Se incendió el 04.08.1971. Vendido para su desguace en diciembre de 1971.

82 El USS Norfolk (DL-1) fue un destructor de la clase homónima. Fue puesto en quilla el 01.09.1949, lanzado el 29.12.1951 y comisionado el 04.03.1953. Descomisionado el 15.01.1970. Dado de baja el 01.11.1973, fue vendido para su desguace el 22.08.1974. 
83 El USS John Willis (DE-1027) fue un destructor de la clase Dealey. Fue puesto en quilla el 05.07.1955, lanzado el 04.02 .1956 y comisionado el 21.02.1957. Descomisionado el 14.07.1972, fue vendido para su desguace el 08.05.1973.

84 El USS Odax (SS-484) fue un submarino de la clase Guppy II. Originalmente fue de clase Tench antes de la modernización GUPPY. Fue puesto en quilla el 04.12.1944, lanzado el 10.04.1945 y comisionado el 11.07.1945. Descomisionado y transferido a la Marinha do Brasil el 08.07.1972 en donde se le denominó Rio de Janeiro (S-13). Fue dado de baja en 16.12.1978 y vendido para desguace el 18.06.1981.

85 El BAP Velarde (CT21) fue una lancha torpedera de la clase Vosper. Fue puesta en quilla en 1963 y comisionada en 1965. Fue transferida al Servicio de Guardacostas en 1975 en donde se le denominó posiblemente Río Ica. Fue dada de baja a mediados de la década de los ochenta.

86 El BAP Santillana (CT22) fue una lancha torpedera de la clase Vosper. Fue puesta en quilla en 1963 y comisionada en 1965. Fue transferida al Servicio de Guardacostas en 1975 en donde se le denominó Río Pativilca (PC225). Fue dada de baja en1999.

87 El BAP De Los Heros (CT23) fue una lancha torpedera de la clase Vosper. Fue puesta en quilla en 1963 y comisionada en 1965. Fue transferida al Servicio de Guardacostas en 1975 en donde se le denominó posiblemente Río Chicama. Fue dada de baja a mediados de la década de los ochenta.

88 El BAP Herrera (CT24) fue una lancha torpedera de la clase Vosper. Fue puesta en quilla en 1963 y comisionada en 1965. Fue transferida al Servicio de Guardacostas en 1975 en donde se le denominó Río Locumba (PC-227). Fue dada de baja a mediados de la década de los ochenta.

89 El BAP Larrea (CT25) fue una lancha torpedera de la clase Vosper. Fue puesta en quilla en 1963 y comisionada en 1965. Fue transferida al Servicio de Guardacostas en 1975 en donde se le denominó posiblemente RÍO HUAURA. Fue dada de baja a mediados de la década de los ochenta.

90 El BAP Sánchez Carrión (CT26) fue una lancha torpedera de la clase Vosper. Fue puesta en quilla en 1963 y comisionada en 1965. Fue transferida al Servicio de Guardacostas en 1975 en donde se le denominó Río Vítor (PC229). Fue dada de baja al parecer en 1985. 
91 El ARA Santa Fe (S-11) fue un submarino de la clase Balao. Originalmente USS Macabi (SS-375) de la armada estadounidense. Fue puesto en quilla el 01.05.1944, lanzado el 19.09.1944 y comisionado el 29.03.1944. Descomisionado el 16.06.1946. Fue transferido a la Armada Argentina el 03.11.1960 en donde se le denominó Santa Fe. Fue dado de baja el 01.09.1971.

92 El ARA Santiago Del Estero (S-12) fue un submarino de la clase Balao. Originalmente USS Lamprey (SS-372) de la armada estadounidense. Fue puesto en quilla el 22.02.1944, lanzado el 18.06.1944 y comisionado el 17.11.1944. Descomisionado el 03.06.1946. Fue transferido a la Armada Argentina el 03.11.1960 en donde se le denominó Santiago Del Estero. Fue dado de baja el 01.09.1971.

93 El BAP Lobitos (ATP159) fue un petrolero de la clase Sechura. Fue puesto en quilla el 27.06.1963, lanzado en mayo de 1965 y comisionado en 1966. Fue dado de baja en 1997.

94 El USS Mac Donough (DDG-39) fue un destructor de la clase Farragut. Fue puesto en quilla el 15.04.1958, lanzado el 09.07.1959 y comisionado el 04.11.1961. Descomisionado el 23.10.1992. Dado de baja el 30.11.1992, fue vendido para su desguace.

95 El USS Bordelon (DDR-881) fue un destructor de la clase Gearing. Fue puesto en quilla el 09.09.1944, lanzado el 03.03.1945 y comisionado el 05.06.1945. Descomisionado el 01.02.1977. Fue transferido a la armada iraní en julio de 1977 para ser canibalizado.

96 El USS Edward Mc Donell (DE-1043) fue un destructor de la clase Garcia. Fue puesto en quilla el 01.04.1963, lanzado el 15.02.1964 y comisionado el 15.02.1965. Descomisionado el 30.09.1988. Dado de baja el 15.12.1992, fue vendido para su desguace el 25.07.1995.

97 El USS Trumpetfish (SS-425) fue un submarino de la clase Guppy II. Originalmente fue de clase Balao antes de la modernización Guppy. Fue puesto en quilla el 23.08.1943, lanzado el 13.05.1945 y comisionado el 29.01.1946. Descomisionado y transferido a la Marinha do Brasil el 15.10.1973 en donde se le denominó Goias (S-15). Fue dado de baja el 16.04.1990.

98 El ARC Almirante Tono (DT-04) fue un destructor de escolta de la clase Buckley. Originalmente USS Bassett (DE-672) de la armada estadounidense. Fue puesto en quilla el 28.11.1943, lanzado el 15.01.1944 y comisionado el 23.02.1945. Descomisionado el 29.04.1946. Nuevamente 
comisionado el 07.12.1950 y descomisionado el 26.11.1957. Fue dado de baja el 01.05.1967. Fue transferido a la armada colombiana el 06.09.1968. Dado de baja a mediados de los años setenta.

99 El ARC Córdoba (DT-15) fue un destructor de escolta de la clase Rudderow. Originalmente USS Ruchamkin (DE-228) de la armada estadounidense. Fue puesto en quilla el 14.02.1944, lanzado el 15.06.1944 y comisionado el 16.09.1945. Descomisionado el 27.02.1946. Nuevamente comisionado el 19.03.1951 y descomisionado el 13.08.1957. Comisionado nuevamente el 18.11.1961. Descomisionado y transferido a la armada colombiana el 24.11.1969. Fue dado de baja en 1980. Desde 2004 es buque museo en el parque Jaime Duque de Bogotá.

100 El BAP Almirante Grau (CLM-81) fue un crucero ligero de la clase De Ruyter. Originalmente HRMS De Ruyter (C-801) de la armada de los Países Bajos. Fue puesto en quilla el 05.09.1939, lanzado el 24.12.1941 y comisionado el 18.11.1953. Descomisionado el 13.10.1972. Fue transferido a la Marina de Guerra del Perú el 23.05.1973. Dado de baja el 26.09.2017. Actualmente se encuentra en espera de destino.

101 El BAP Independencia (ATA-130) fue un transporte de la clase Arcturus. Originalmente USS Bellatrix (AKA-3) y Raven (AK-20) de la armada estadounidense. Fue puesto en quilla el 20.11.1940, lanzado el 15.08.1941 y comisionado el 17.02.1942. Descomisionado el 01.04.1946. Nuevamente comisionado el 16.05.1952 y descomisionado el 13.08.1957. Transferido a la Marina de Guerra del Perú el 20.07.1963. Fue dado de baja en octubre de 1991. Vendido para su desguace, se hundió rumbo a Japón en 1992.

102 El BAP Paita (LT-35) fue un buque de desembarco de la clase LST-491. Originalmente USS Burnett County (LST-512) de la armada estadounidense. Fue puesto en quilla el 22.07.1943, lanzado el 10.12.1943 y comisionado el 08.01.1944. Descomisionado el 28.03.1947. Fue dado de baja el 18.02.1957. Transferido a la Marina de Guerra del Perú el 11.10.1957. Fue dado de baja en 1983.

103 Ver el capítulo correspondiente a esta nave en el presente trabajo.

104 Ver el capítulo correspondiente a esta nave en el presente trabajo.

105 El BAP Arica (SS-36) es un submarino del Tipo 209/1100. Fue puesto en quilla el 01.10.1971, lanzado el 05.04.1974 y comisionado el 21.01.1975. Actualmente en servicio. 
106 El BAP Islay (SS-35) es un submarino del Tipo 209/1100. Fue puesto en quilla el 15.03.1971, lanzado el 11.10.1973 y comisionado el 29.08.1974. Actualmente en servicio.

107 El BAP CHIMBOTE (LT-34) fue un buque de desembarco de la clase LST-1. Originalmente USS LST-283 de la armada estadounidense. Fue puesto en quilla el 02.08.1943, lanzado el 10.10.1943 y comisionado el 18.01.1943. Descomisionado el 13.06.1946. Fue vendido a la compañía Northrup H. Castle de Hawai para su conversión a servicio comercial que lo denominó M/S Rawhiti. Transferido a la Marina de Guerra del Perú el 21.12.1951. Fue dado de baja en 1984.

108 El BAP La Pedrera (S-49) fue un submarino de la clase Guppy IA. Originalmente USS Sea Poacher (SS-406), fue de clase Balao antes de la modernización GUPPY. Fue puesto en quilla el 23.02.1944, lanzado el 20.05.1944 y comisionado el 31.07.1944. Descomisionado el 15.11.1969. Dado de baja el 15.08.1973. Transferido a la Marina de Guerra del Perú el 01.07.1974 en donde se le denominó previamente Pabellón de Pica. Fue dado de baja el 30.04.1992.

109 El BAP Carvajal (FM-51) es una fragata de la clase Lupo. Fue puesta en quilla el 08.08.1974, lanzada el 17.11.1976 y comisionada el 23.12.1979. Fue transferida al Servicio de Guardacostas el 26.12.2013 en donde se le denominó Guardiamarina San Martin y reclasificado como patrullero oceánico (PO-201). Actualmente en servicio.

110 Ver el capítulo correspondiente a esta nave en el presente trabajo.

111 El BAP Zorritos (ATP158) fue un petrolero de la clase Sechura. Fue puesto en quilla en 1955, lanzado el 26.07.1958 y comisionado en 1959. Fue dado de baja en 1999.

\section{REFERENCIAS BIBLIOGRÁFICAS}

Buenaño Muro, J., COSIO, J. C., GAMBETTA, N., GAMIO PALACIO, F., MARTIN, J. C., PASTOR, A. E. y SORIANO, A. L. (1982). Héroes y marinos notables. Apuntes biográficos. Vol I. Lima: Museo Naval del Perú, Dirección General de Intereses Marítimos.

CRAWFORD, S. (2002). Portaaviones y acorazados. Madrid: Editorial LIBSA. 
COSIO, J. C. (1982). Capitán de Corbeta Elías Aguirre Romero. Lima: Ministerio de Marina, Dirección General de Intereses Marítimos, Museo Naval del Perú.

Instituto Idrográfico de La Marina (1988). Almanacco Navale 1988. Génova: Tipo Litografia dell'Instituto Idrografico della Marina, Génova, Italia.

Jackson, R. (2002). Destructores, fragatas y corbetas. Madrid: Editorial LIBSA.

Martin, J. C. (1982). Teniente Primero José Melitón Rodríguez. Lima: Ministerio de Marina, Dirección General de Intereses Marítimos, Museo Naval del Perú.

Moore, J. (1987). Janes's Fighting Ships 1987-88. Londres: Jane's Information Group, $91^{\circ}$ Edición.

Ortiz Sotelo, J. (2001). Apuntes para la historia de los submarinos peruanos. Lima: Fondo Editorial de la Biblioteca Nacional del Perú.

Preston, A. (1998). Jane's Fighting Ships of World War II. Nueva York: Crescent Books.

Preston, A. (1999). The World's Great Aircraft Carriers. Londres: Editorial Chancellor Books.

Rodríguez Asti, J. (2000). Cruceros, buques de la Marina de Guerra del Perú desde 1894. Lima: Fondo de Publicaciones de la Dirección de Intereses Marítimos.

Sharpe, R. (2000). Jane's Fighting Ships 2000-2001. Londres: Jane's Information Group, $103^{\circ}$ Edición.

\section{FUENTES ELECTRÓNICAS}

www.archive.hnsa.org

www.bangust.com

buquesarmadaperu.blogspot.pe

ericknavasmodelismonaval.blogspot.pe

www.hazegray.com

www.histamar.com

www.history.navy.mil 
www.marina.mil.pe

www.marinha.mil.br

www.microworks.net

www.naval.com.br

www.navsouce.org

www.navypedia.org

www.netmarine.net

www.peru.justia.com

www.pilotoviejo.com

www.podermilitar.blogspot.pe

www.promo1961 esnaperu.info

www.pwencycl.kgbudge.com

www.remolcadoresuruguayos.blogspot.pe

www.uboat.net

www.ussslater.org 